\title{
Evaluation de la mise en oeuvre et des réalisations de l'Approche 3D au sein du Plan d'Action National de Planification Familiale (PANPF) au Sénégal-Partie 1: Revue Documentaire
}

\author{
Leslie Dubent \\ Population Council \\ Fatou Mbow \\ Population Council \\ Nafissatou Diop \\ Population Council \\ Fatou Ndiaye Turpin \\ Bocar Mamadou Daff
}

See next page for additional authors

Follow this and additional works at: https://knowledgecommons.popcouncil.org/departments_sbsr-rh

Part of the Demography, Population, and Ecology Commons, Family, Life Course, and Society Commons, International Public Health Commons, Maternal and Child Health Commons, and the Women's Health Commons How does access to this work benefit you? Let us know!

\section{Recommended Citation}

Dubent, Leslie, Fatou Mbow, Nafissatou Diop, Fatou Ndiaye Turpin, Bocar Mamadou Daff, and Babacar Mane. 2015. "Evaluation de la mise en oeuvre et des réalisations de l'Approche 3D au sein du Plan d'Action National de Planification Familiale (PANPF) au Sénégal-Partie 1: Revue Documentaire." Dakar: Population Council, The Evidence Project. 


\section{Authors}

Leslie Dubent, Fatou Mbow, Nafissatou Diop, Fatou Ndiaye Turpin, Bocar Mamadou Daff, and Babacar Mane 


\section{Evaluation de la mise en œuvre et des}

\section{réalisations de l'Approche 3D au sein du Plan d'Action National de Planification} Familiale (PANPF) au Sénégal

\section{PARTIE 1 : REVUE DOCUMENTAIRE}

\section{Leslie Dubent, Assistante de Recherche, Population Council}

Fatou Bintou Mbow, Chargée de Programmes, Population Council Nafissatou Diop, Country Director, Population Council

Fatou Ndiaye, Maternal and Child Health/Family Planning Specialist, USAID Senegal Bocar Mamadou Daff, Directeur, Direction de la Santé de la Reproduction et de la Survie de l'Enfant. Ministère de la Santé et de l'Action Sociale du Sénégal Babacar Mané, Chargé de Programmes, Population Council

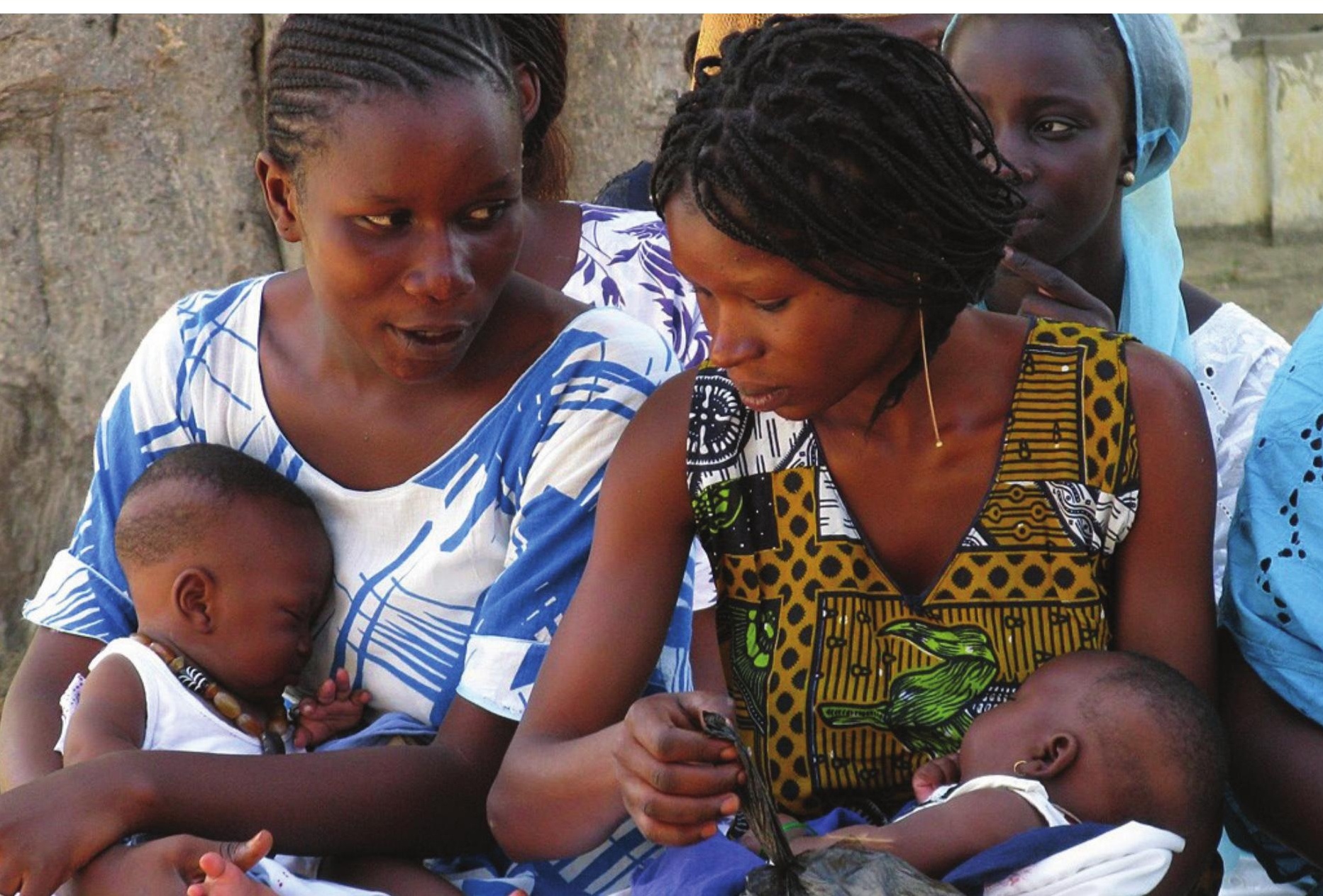




\section{The Evidence Project}

Population Council

4301 Connecticut Avenue, NW, Suite 280

Washington, DC 20008 USA

tel +12022379400

evidenceproject.popcouncil.org

Le Projet Evidence est rendu possible grâce au généreux support du Peuple Américain à travers l'Agence des Etats Unis pour le Développement International (USAID) sous les termes de l'accord de coopération no. AID-OAA-A-13-00087. Le contenu de ce document est la seule responsabilité du Projet Evidence et ne reflète pas nécessairement les points de vue de l'USAID ou du Gouvernement des Etats Unis.

\section{Evidence}

Le Projet Evidence utilise la science de la mise en œuvre -la production, traduction, et utilisation stratégique d'évidences - pour renforcer et mettre à l'échelle des programmes de planification familiale et de santé de la reproduction afin de réduire les grossesses non désirées partout dans le monde. Le Projet Evidence est dirigé par le Population Council en partenariat avec le réseau INDEPTH, International Planned Parenthood Federation, PATH, Population Reference Bureau, et un réseau de ressources universitaires.

Publié dans Décembre 2015.

Suggested citation: Dubent L, Mbow FB, Diop N, Ndiaye F, Daff BM \& Mané B. 2015. "Evaluation de la mise en oeuvre et des réalisations de l'Approche 3D au sein du Plan d'Action National de Planification Familiale (PANPF) au Sénégal. Partie 1: Revue dela littérature," Rapport. Dakar, Sénégal: Population Council, Le Projet Evidence.

Crédit photo sur la page de garde (C) 2011 Adrienne Allison World Vision, Courtesy of Photoshare

(C) 2015 The Population Council, Inc. 


\section{Table des matières}

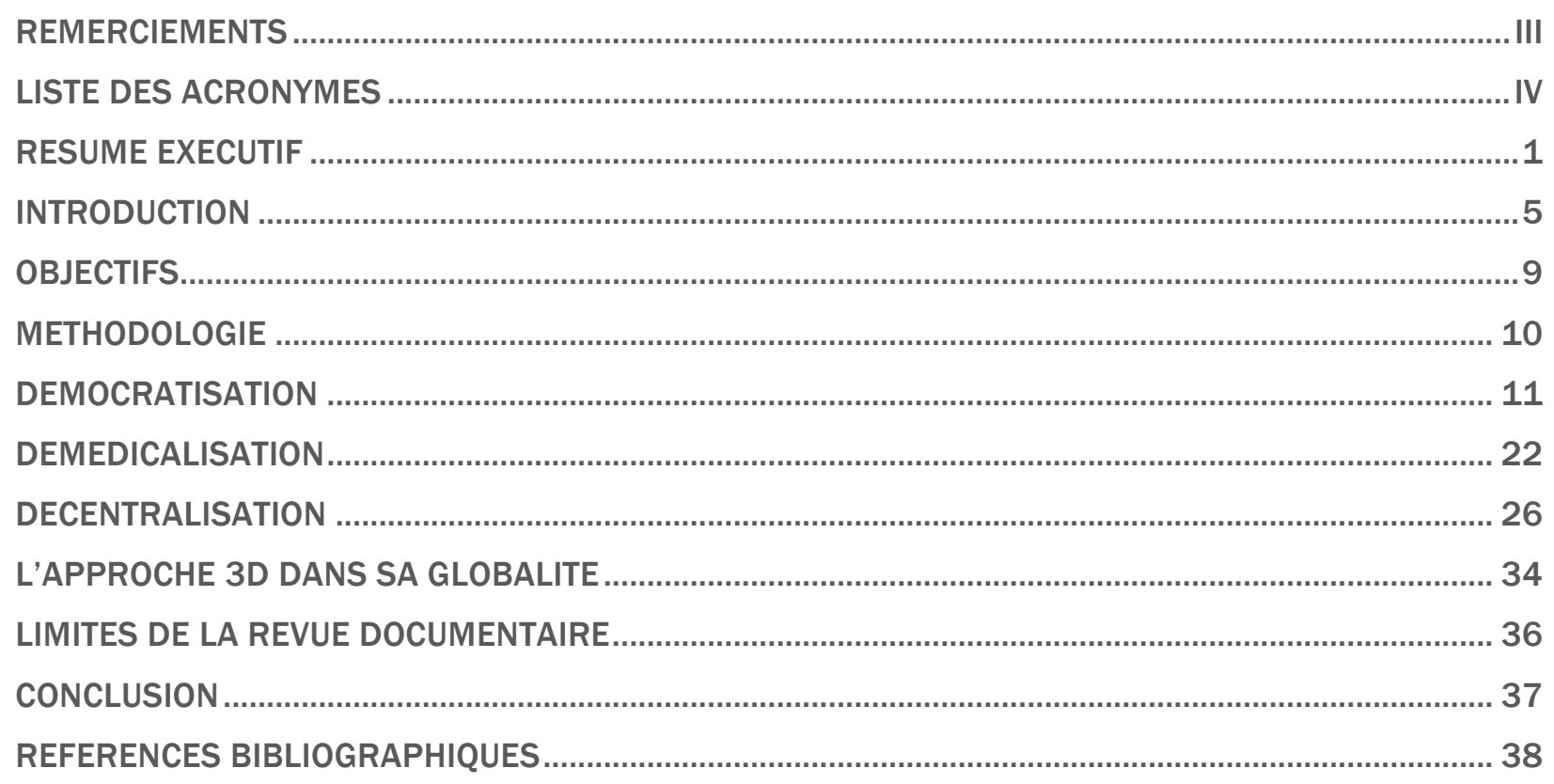




\section{Remerciements}

Les auteurs remercient toutes les institutions, organisations et personnes qui ont contribué à la réalisation de cette étude en facilitant l'accès à la documentation disponible ou en orientant vers d'autres parties prenantes susceptibles de détenir de la documentation utile. 


\section{Liste des acronymes}

\begin{tabular}{|c|c|c|c|}
\hline $3 \mathrm{D}$ & $\begin{array}{l}\text { Démocratisation, } \\
\text { Démédicalisation, }\end{array}$ & MLDA & $\begin{array}{l}\text { Méthodes à Longue Durée } \\
\text { d'Action }\end{array}$ \\
\hline & Décentralisation & MLE & Measurement, Learning and \\
\hline AcDev & Action et Développement & & Evaluation \\
\hline ADEMAS & $\begin{array}{l}\text { Agence pour le Développement } \\
\text { du Marketing Social }\end{array}$ & MSAS & $\begin{array}{l}\text { Ministère de la Santé et de } \\
\text { l'Action Sociale }\end{array}$ \\
\hline ANSFES & $\begin{array}{l}\text { Association des Sages-Femmes } \\
\text { d'Etat du Sénégal }\end{array}$ & $\begin{array}{l}\text { MSI } \\
\text { ODD }\end{array}$ & $\begin{array}{l}\text { Marie Stopes International } \\
\text { Objectifs de Développement }\end{array}$ \\
\hline ASBEF & $\begin{array}{l}\text { Association Sénégalaise pour le } \\
\text { Bien-Etre Familial }\end{array}$ & OII & $\begin{array}{l}\text { Durable } \\
\text { Offre Initiale d'Injectable }\end{array}$ \\
\hline ASC & Agent de Santé Communautaire & OIP & Offre Initiale de Pilule \\
\hline BNS & Besoins Non Satisfaits & OMD & Objectifs du Millénaire pour le \\
\hline CEFOREP & $\begin{array}{l}\text { Centre de Formation et de } \\
\text { Recherche en Santé de la } \\
\text { Reproduction }\end{array}$ & ONG & $\begin{array}{l}\text { Développement } \\
\text { Organisation Non- } \\
\text { Gouvernementale }\end{array}$ \\
\hline CIPD & $\begin{array}{l}\text { Conférence Internationale sur la } \\
\text { Population et le Développement }\end{array}$ & PANPF & $\begin{array}{l}\text { Plan d'Action National de } \\
\text { Planification Familiale }\end{array}$ \\
\hline DBC & $\begin{array}{l}\text { Distribution à Base } \\
\text { Communautaire }\end{array}$ & $\begin{array}{l}\text { PF } \\
\text { PHI }\end{array}$ & $\begin{array}{l}\text { Planification Familiale } \\
\text { Pratique à Haut Impact }\end{array}$ \\
\hline $\begin{array}{l}\text { DIU } \\
\text { DMPA-IM }\end{array}$ & $\begin{array}{l}\text { Dispositif Intra-Utérin } \\
\text { Dépôt d'Acétate } \\
\text { Médroxyprogestérone - Forme } \\
\text { Intramusculaire }\end{array}$ & PNDS & $\begin{array}{l}\text { Pharmacie Nationale } \\
\text { d'Approvisionnement } \\
\text { Programme National de } \\
\text { Développement Sanitaire }\end{array}$ \\
\hline DMPA-SC & $\begin{array}{l}\text { Dépôt d'Acétate } \\
\text { Médroxyprogestérone - Forme } \\
\text { Sous-Cutanée }\end{array}$ & $\begin{array}{l}\text { PPP } \\
\text { PPS } \\
\text { PRA }\end{array}$ & $\begin{array}{l}\text { Partenariat Public Privé } \\
\text { Point de Prestation de Services } \\
\text { Pharmacie Régionale }\end{array}$ \\
\hline DSRSE & $\begin{array}{l}\text { Direction de la Santé de la } \\
\text { Reproduction et de la Survie de } \\
\text { l'Enfant }\end{array}$ & PTF & $\begin{array}{l}\text { d'Approvisionnement } \\
\text { Partenaires Techniques et } \\
\text { Financiers }\end{array}$ \\
\hline ECD & Equipe Cadre de District & RSJ & Réseau Siggil Jigeen \\
\hline ECR & Equipe Cadre de Région & SAFI & Sages-Femmes Itinérantes \\
\hline EDS & $\begin{array}{l}\text { Enquête Démographique et de } \\
\text { Santé }\end{array}$ & SMNI & $\begin{array}{l}\text { Santé Maternelle, Néonatale et } \\
\text { Infantile }\end{array}$ \\
\hline EDS-C & $\begin{array}{l}\text { Enquête Démographique et de } \\
\text { Santé Continue }\end{array}$ & $\begin{array}{l}\text { SR } \\
\text { SRAJ }\end{array}$ & $\begin{array}{l}\text { Santé de la Reproduction } \\
\text { Santé de la Reproduction des }\end{array}$ \\
\hline EDS-MICS & $\begin{array}{l}\text { Enquête Démographique et de } \\
\text { Santé à Indicateurs Multiples }\end{array}$ & TPC & $\begin{array}{l}\text { Adolescents et des Jeunes } \\
\text { Taux de Prévalence }\end{array}$ \\
\hline FHI & Family Health International & Contraceptive & \\
\hline GEEP & $\begin{array}{l}\text { Groupe pour l'Etude et } \\
\text { l'Enseignement de la Population }\end{array}$ & UNFPA & $\begin{array}{l}\text { Fonds des Nations Unies pour la } \\
\text { Population }\end{array}$ \\
\hline ICP & Infirmier Chef de Poste & USAID & Agence des Etats Unis pour le \\
\hline IPM & Informed Push Model & & Développement International \\
\hline ISSU & $\begin{array}{l}\text { Initiative Sénégalaise de Santé } \\
\text { Urbaine }\end{array}$ & & \\
\hline
\end{tabular}




\section{Résumé exécutif}

L'approche 3D, acronyme pour Démocratisation, Démédicalisation et Décentralisation, aurait été présentée par le Sénégal pour la première fois en Afrique de l'Ouest francophone lors de la conférence "Population, développement et planification familiale en Afrique de l'ouest francophone : l'urgence d'agir" en février 2011. Il s'agit aussi de la stratégie qui sous-tend le Plan d'Action National de Planification Familiale 2012-2015. A l'heure de la préparation d'un nouveau plan d'action, la documentation de l'approche est essentielle.

Objectifs et méthodologie

Les objectifs de la revue documentaire sont de :

- Déterminer la quantité de documentation existante et disponible,

- Identifier les personnes à contacter et solliciter leur participation à des interviews planifiées dans la deuxième phase de l'étude,

- Eviter la duplication de ce qui peut avoir déjà été documenté et mettre l'accent sur les domaines qui nécessitent plus d'attention pour la documentation.

La revue documentaire a été effectuée en faisant appel aux parties prenantes investies dans au moins l'un des aspects de l'approche 3D.

\section{Démocratisation}

L'environnement politique a largement favorisé la démocratisation de la planification familiale (PF) ces dernières années, notamment en augmentant les ressources financières et en renforçant le partenariat publicprivé. Les problèmes liés à l'accès financier ont été traités en partie en fixant le prix des contraceptifs distribués dans les structures publiques de santé. Les barrières socioculturelles et celles relatives à l'accès géographique ont été en partie levées en renforçant la communication visant essentiellement les hommes, en impliquant les leaders religieux, en mettant en place des programmes de sensibilisation et de distribution à base communautaire, en recourant au leadership des femmes (programme «Bajenu Gox »), et en menant des actions ciblant les adolescents et les jeunes.

\section{Démédicalisation}

La distribution à base communautaire de la PF s'est largement développée ces dernières années permettant d'atteindre des femmes vivant dans des zones défavorisées et/ou enclavées et de répondre à leurs besoins jusque-là non satisfaits. Les activités de délégation des tâches se sont aussi élargies permettant notamment au personnel communautaire d'injecter les contraceptifs intramusculaires et sous-cutanés.

\section{Décentralisation}

La décentralisation de la PF consiste en l'implication de tous les niveaux de la pyramide sanitaire et en l'extension des initiatives à toutes les zones. On peut citer par exemple le passage à l'échelle de l'offre initiale de pilule, de l'offre initiale d'injectables, ou l'amélioration de la disponibilité des produits par l'Informed Push Model (IPM), les Pharmacies Régionales d'Approvisionnement mobiles et le projet «Jegesi naa ».

Les différents niveaux de la pyramide sanitaire (régions, districts,...) sont plus impliqués dans la définition des objectifs et responsabilisés. Les collectivités locales commencent également à être de plus en plus impliquées financièrement. 


\section{L'Approche 3D dans sa globalité}

Démocratisation, démédicalisation et décentralisation ont beaucoup avancé ces dernières années au Sénégal. Cependant, des barrières socio-culturelles perdurent, limitant encore parfois la démocratisation et l'accès à la PF. La démédicalisation est en pleine expansion et les projets initiés devront être passés à l'échelle. De nouveaux projets devront aussi être mis en œuvre afin de permettre un accès équitable à la PF à toutes les femmes qui le désirent. Enfin, la décentralisation permet l'extension de projets prometteurs tels que l'IPM ou l'introduction du Sayana ${ }^{\circledR}$ Press, mais elle doit encore faire face à des défauts d'implication et de financements de diverses parties prenantes.

Les 3D sont très liés les uns aux autres et juger de leur progression individuelle n’est pas chose aisée.

L'ensemble des activités liées à la démocratisation, à la démédicalisation et à la décentralisation de la PF ont permis une nette amélioration des indicateurs dont le Taux de Prévalence Contraceptive passé de 12\% à 20\% entre l' EDS-MICS de 2010-2011 et l'EDS-C de 2014. Cependant les efforts devront perdurer et porter d'une part sur la recherche de financements innovants et sur l'implication financière accrue des diverses parties prenantes, et d'autre part sur des actions ciblant les groupes importants que sont les hommes, les leaders religieux et les adolescents/jeunes. L'approche multisectorielle devra elle aussi être plus soutenue et approfondie.

\section{Limites de la revue documentaire}

L'approche 3D est jusqu'ici très peu documentée. L'accès aux documents relatifs à l'approche même ou aux activités s'y rapportant a été compliqué. Cette revue documentaire s'est attachée à décrire au mieux les différentes composantes de l'approche 3D en PF mais n'est cependant pas exhaustive.

De plus, il a parfois été difficile de vérifier dans quelle mesure les promesses de financement sont tenues et à quel degré les actions décrites sont implémentées en pratique.

\section{Conclusion}

Le Sénégal a connu des progrès indéniables dans le domaine de la PF ces dernières années et notamment depuis le lancement du PANPF en 2012, dont les actions stratégiques reposent sur les concepts de démocratisation, démédicalisation et décentralisation. Cette approche 3D devra donc être développée et démocratisée pour une pérennisation, notamment au sein du prochain plan. 


\section{Executive Summary (English)}

The 3D approach, which stands for democratization, de-medicalization, and decentralization, was presented by Senegal for the first time in Francophone West Africa at the conference "Population, Development and Family Planning in Francophone West Africa: The urgency to act," in February 2011. This is also the strategy behind the National Family Planning Action Plan 2012-2015. As the time for preparing a new action plan comes, documentation of the 3D approach is essential.

\section{Objectives and methods}

The objectives of the desk review are:

- To determine the amount of literature existing and available;

- To identify contact persons and solicit their participation in interviews planned for the second phase of the study;

- To avoid duplication of what may have already been documented and focus on areas that need more attention for documentation.

The desk review was conducted by calling on stakeholders invested in at least one aspect of the 3D approach.

\section{Democratization}

The policy environment heavily emphasized the democratization of family planning (FP) in recent years, including increasing financial resources and strengthening public-private partnerships. Problems related to financial access have been addressed partly by setting the price of contraceptives dispensed in public health facilities. Socio-cultural barriers and those related to geographical access were partially overcome by strengthening communication, mainly directed towards men, and by involving religious leaders, implementing awareness programs and community-based distribution programs, capitalizing on women's leadership (e.g. the "Bajenu Gox" program), and conducting activities directed towards adolescents and youth.

\section{De-medicalization}

Community-based distribution of FP has developed considerably in recent years, expanding the reach of services to women living in disadvantaged and/or isolated areas, and better responding to their previously unmet needs. Task shifting activities were also expanded, particularly by allowing community level staff to perform injections of intramuscular and subcutaneous contraceptives.

\section{Decentralization}

FP decentralization consists of involving all levels of the health pyramid and extending projects to all areas. Examples are the scaling up of initial supplies of pills and injectables, and the improvement of product availability through the Informed Push Model (IPM), mobile Regional Supply Pharmacies, and "Jegesi naa" programs.

The different levels of the health pyramid (regions, districts, and so forth) are more involved in setting objectives and are more empowered. Local governments are also increasingly involved financially.

\section{The 3D approach as a whole}

Democratization, De-medicalization, and Decentralization have progressed considerably in recent years in Senegal. However, socio-cultural barriers persist, and sometimes still limit Democratization and access to FP. 
De-medicalization is expanding, and existing projects should be scaled up. New projects also must be implemented to allow equitable access to FP for all women who want it. Finally, Decentralization allows the expansion of promising programs such as IPM from the ISSU project or the introduction of Sayana ${ }^{\circledR}$ Press, but it still faces lack of involvement and funding from various stakeholders.

The three Ds are very related to each other and assessing their individual contributions is not easy.

All activities related to Democratization, De-medicalization and Decentralization of FP led to a marked improvement in indicators, including the Contraceptive Prevalence Rate (CPR), which increased from 12 percent to 20 percent between 2010-2011 and 2014. However, efforts must be continued and focus on finding innovative financing options and increasing financial involvement of various stakeholders, and also on activities directed towards key groups, specifically men, religious leaders and adolescents/youth. The multi-sectoral approach should also be sustained and expanded.

\section{Limit of the desk review}

The 3D approach has not been extensively documented so far. Access to documents about the approach itself or related activities was complicated. This desk review sought to describe as well as possible the various components of the 3D approach in the FP context, but it is not exhaustive.

Furthermore, it was sometimes difficult to ascertain the extent to which funding promises have been kept and the extent to which described actions have been implemented in practice.

\section{Conclusion}

Senegal has made undeniable progress in the field of FP in recent years, particularly since the launch of the NFPAP in 2012, whose strategic actions are based on the concepts of Democratization, De-medicalization, and Decentralization. This 3D approach should be developed and generalized for sustainability, particularly within the next plan. 


\section{Introduction}

Le Sénégal, comme la plupart des pays d'Afrique de l'Ouest francophone, connait encore des taux de fécondité et de mortalité maternelle et infantile élevés. Afin de réduire ces taux et atteindre les objectifs nationaux et internationaux dans le domaine de la santé, et notamment les Objectifs du Millénaire pour le Développement (OMD) et plus récemment les Objectifs de Développement Durable (ODD), le Sénégal a entrepris divers chantiers. En particulier, il a choisi de faire de la planification familiale (PF) une priorité nationale, sachant que la PF est l'une des stratégies les plus efficientes pour améliorer la santé de la femme et de l'enfant, et qu'au-delà de ses bénéfices sanitaires directs, elle contribue également au développement économique et social et à l'autonomisation des femmes.

Les efforts pour améliorer le contexte de la Santé de la Reproduction (SR), et en particulier la promotion de la PF, datent de plusieurs décennies au Sénégal. Les paragraphes suivants en retracent l'historique afin de mettre en contexte le Plan d'Action National pour la Planification Familiale 2012-2015 (PANPF 2012-2015) et l'approche 3D, avant de documenter la contribution de l'approche à l'atteinte des objectifs du PANPF.

\section{HISTORIQUE DES PROGRAMMES DE PF}

C’est en 1970 que les services de PF ont été introduits au Sénégal, à la clinique de la Croix Bleue, établissement privé de Dakar. En 1975, la première clinique de l'Association Sénégalaise pour le Bien-Etre Familial (ASBEF) a vu le jour, offrant également des services PF (Sidze, Lardoux, Speizer et al., 2015 ; Mané, Diop, Termini et al., 2012 ; Mané, 1994).

Cependant, jusqu'en 1980 la promotion des contraceptifs était interdite au Sénégal par une loi française datant de 1920. Après l'abrogation de cette loi, la volonté des pouvoirs publics à promouvoir la PF s'est manifestée à travers la mise en œuvre du «Projet Population et Santé de la Famille ", financé par l'Agence des Etats Unis pour le Développement International (USAID), puis le « Projet de bien-être familial » financé par le Fonds des Nations Unies pour la Population (UNFPA) (Sidze et al., 2015 ; Mané et al., 2012).

Dans la poursuite des efforts de promotion de la PF, le Gouvernement du Sénégal a adopté en 1988 la Déclaration de Politique de la Population qui réaffirme sa volonté de donner à la PF une place centrale dans les programmes de développement en général et de santé en particulier. Ainsi, le Sénégal fut le premier pays de la sous-région à élaborer et adopter une politique de population reposant sur les principes d'une diminution du rythme de la croissance démographique (Sidze et al., 2015).

En 1991, le Programme National de Planification Familiale a été lancé. En 1997, afin de se conformer aux recommandations de la Conférence Internationale sur la Population et le Développement (CIPD) dont le programme d'action a été adopté en 1994, le Gouvernement du Sénégal a créé le Service National de la Santé de la Reproduction pour rehausser l'ancrage institutionnel du programme national de PF (Daff, 2011).

La loi relative à la SR, adoptée et promulguée en aout 2005, vise à lever les obstacles et contraintes identifiés et à permettre l'accès aux contraceptifs à toutes les femmes en âge de procréer (Gouvernement du Sénégal, 2005).

En 2002, la Division de la Santé de la Reproduction est créée au sein de la Direction de la Santé. Cette Division deviendra en 2012 la Direction de la Santé de la Reproduction et de la Survie de l'Enfant (DSRSE) (Daff, 2011 ; Mané et al., 2012). Sous la coordination de la DSRSE, un Plan d'Action National pour la Planification Familiale (PANPF) a été élaboré et adopté en 2012. Ce plan s’articule autour des orientations du Plan Stratégique de la Santé de la Reproduction 2012-2015 et intègre dans sa mise œuvre celles inscrites dans les documents 
stratégiques antérieurs, notamment la «Feuille de route multisectorielle pour accélérer la réduction de la mortalité et morbidité maternelles et néonatales au Sénégal pour la période 2006-2015 ». En vue d'opérationnaliser le PANPF et d'en atteindre ses objectifs, d'autres documents stratégiques ont été élaborés :

- Les documents de Politiques, Normes et Protocoles (PNP) en Santé de la Reproduction (en cours de révision),

- Le Plan Stratégique de Sécurisation des Produits SR/PF 2011-2015,

- Le Plan Stratégique de Santé Sexuelle et de Reproduction des Adolescent(e)s/Jeunes (SRAJ) 20142018,

- Le Plan National Stratégique de Santé Communautaire 2014-2018,

- Le Plan Opérationnel de Plaidoyer sur la Planification Familiale 2013- 2015.

Tous ces documents intègrent les stratégies du PANPF et se réfèrent aux orientations stratégiques du Plan National de Développement Sanitaire (PNDS) couvrant la période 2009-2018, qui demeure l'instrument de référence en matière de politique sanitaire.

\section{EVOLUTION DES INDICATEURS SR/PF}

L'Indice Synthétique de Fécondité (ISF) au Sénégal a eu tendance à se stabiliser autour de 5,0 enfants par femme au cours de ces dernières années. Cependant, durant cette même période, on a pu observer une diminution progressive des indicateurs de mortalité maternelle et infantile, dont les taux sont passés, entre les Enquêtes Démographiques et de Santé à Indicateurs Multiples (EDS-MICS) de 2005 et de 2010-20111, de 401 à 392/100 000 naissances vivantes pour la mortalité maternelle, de 36 à 34/1 000 pour la mortalité néonatale, et de 121 à 54/1 000 pour la mortalité infanto-juvénile (Ndiaye et Ayad, 2006 ; ANSD et ICF international, 2012, ANSD et ICF International 2015). Toutefois, malgré ces efforts, l'amélioration reste encore insuffisante si l'on se réfère aux OMD 4 et 5 qui visaient à réduire de deux tiers le taux de mortalité des enfants de moins de cinq ans et de trois quarts le taux de mortalité maternelle. En effet, en 2013, le taux de mortalité maternelle diminuait en moyenne de 1,6\% annuellement alors qu'une diminution de 5,5\% est nécessaire pour atteindre l'objectif (Bureau de l’OMS au Sénégal, 2013).

L'évolution du Taux de Prévalence Contraceptive (TPC) n'a pas suivi le même rythme d'évolution. Or, même si l'imputabilité de l'amélioration des indicateurs est difficile à déterminer, le TPC reste un bon indicateur de la réussite des programmes de PF. Le TPC est resté globalement bas jusqu'en 2012 : chez les femmes en union, le TPC pour les méthodes modernes est passé de $8 \%$ en 1997, à $10 \%$ en 2005, et $12 \%$ en 2010-2011. Ce n'est que depuis 2012 que le TPC a connu une augmentation significative, par bonds de quatre points, passant à 16\% en 2013, puis à 20\% en 2014. (ANSD et ICF International, 2012; ANSD et ICF International, 2013 ; ANSD et ICF International, 2015).Cependant, malgré cette importante hausse du TPC, l'EDS continue (EDS-C) de 2014 rapporte un taux de Besoins Non Satisfaits (BNS) en contraception de 25,6\% parmi les femmes en union dont $19,2 \%$ qui souhaitaient différer leur prochaine naissance d'au moins deux ans et 6,3\% qui ne désiraient plus de grossesse du tout, sans toutefois utiliser une méthode contraceptive (ANSD et ICF International, 2015).

Chez les adolescents et les jeunes, qui constituent une cible privilégiée des programmes de SR/PF, notamment de par leur rôle dans le dividende démographique, il a été également noté une évolution du TPC pour les méthodes modernes. Cet indicateur est passé de 1,9\% en 2010-2011 à 12,3\% en 2014 pour les 15-19 ans et de $6,0 \%$ à 12,2\% pour les 20-24 pour la même période. (ANSD et ICF International, 2012 ; ANSD et ICF International, 2015).

$1 \quad$ Les taux de mortalités maternelle et infantile ne sont pas disponibles dans les rapports plus récents des EDS Continues de 20122013 et de 2014 
Cette nette amélioration du TPC a coïncidé avec la mise en œuvre du PANPF 2012-2015. Ces progrès ont pu être réalisés grâce à la volonté manifeste des autorités publiques de repositionner le programme national de PF et d'asseoir sa mise en œuvre sur l'approche des 3D, à savoir la Démocratisation, la Démédicalisation et la Décentralisation.

\section{NAISSANCE ET DEFINITION DE L'APPROCHE 3D}

En Février 2011, la conférence « Population, Développement et Planification Familiale en Afrique de l'Ouest: L'Urgence d'Agir » a réuni à Ouagadougou, Burkina Faso, les représentants de huit pays de l'Afrique de l'Ouest francophone (Bénin, Burkina Faso, Guinée, Mali, Mauritanie, Niger, Sénégal et Togo) et leurs partenaires techniques et financiers (PTF). La Côte d'Ivoire a rejoint l'initiative plus tard. Lors de la conférence, les états se sont accordés sur la nécessité de prendre des mesures concrètes afin d'augmenter l'utilisation de la PF dans leurs pays respectifs. C’est ainsi que le Partenariat de Ouagadougou est né (Partenariat de Ouagadougou, 2011).

Au cours de cette conférence, le Sénégal a présenté une approche novatrice pour structurer les interventions de PF appelée l'approche 3D². Le Sénégal applique cette approche non seulement dans le domaine de la PF mais aussi pour d'autres interventions en matière de santé, notamment celles portant sur la santé maternelle, néonatale et infantile, la lutte contre le paludisme et la nutrition (Daff, 2011).

Pour la PF, à travers l'approche 3D :

- La «Démocratisation » est conçue pour faciliter l'accès aux services par le biais d'une approche multisectorielle et participative;

- La «Démédicalisation » vise à permettre à des agents non médicaux de fournir une gamme variée de services PF aux populations vulnérables (rurales, urbaines défavorisées, pauvres et jeunes), notamment par le biais d'une distribution à base communautaire;

- La «Décentralisation » vise à renforcer les systèmes de santé aux niveaux régional, district et communautaire, tout en renforçant la bonne gouvernance, la responsabilisation et la collaboration intersectorielle.

L'approche 3D constitue le socle de la mise en œuvre du PANPF.

\section{LE PLAN D'ACTION NATIONAL DE PLANIFICATION FAMILIALE (PANPF) 2012-2013}

Suite à la conférence de Ouagadougou, le Sénégal a été l'un des premiers pays du Partenariat à élaborer un plan national PF. Le PANPF 2012-2015 a pour objectif principal d'offrir à toutes les femmes du Sénégal un accès égal et de qualité aux services de PF. Il a été lancé le 28 Novembre 2012, et ambitionne d'augmenter le TPC pour les femmes en union des 12\% reportés dans l'EDS 2010-2011 à 27\% en 2015. Cet objectif nécessite le recrutement de 350000 nouvelles utilisatrices de méthodes de contraception, dont la contribution se répartit en : 125000 nouvelles clientes par le secteur public, 100000 par le secteur privé, et 125000 par le niveau communautaire. Le plan prévoit aussi de réduire les BNS d'environ 30\% à 15\% (DSR et MSAS, 2012).

Le PANPF est constitué d'actions stratégiques couvrant six domaines d'intervention, qui, eux-mêmes, reposent sur les $3 \mathrm{D}$ :

- La communication au niveau national

2 L'approche 3D avait été présentée précédemment lors d'une conférence à Kigali au Rwanda en 2010 mais aucun document attestant cette information n'a pu être retrouvée. 
- Le plaidoyer ciblé

- La disponibilité des produits

- L'extension de l'offre à base communautaire

- Le renforcement de l'offre privée

- L'amélioration de l'offre publique 


\section{Objectifs}

Le PANPF se terminant en 2015, le Ministère de la Santé et de l'Action Sociale (MSAS), à travers la DSRSE, s'est engagé à l'élaboration d'un nouveau plan. Aussi, le moment est idéal pour documenter la mise en œuvre et les réalisations de l'approche $3 \mathrm{D}$ sur laquelle reposait sa mise en ouvre et de fournir les évidences qui pourraient contribuer à la bonne préparation du prochain plan. De plus, compte tenu du rôle de leader du Sénégal en ce qui concerne la PF en Afrique de l'Ouest francophone, cette étude permettra aussi de tirer les leçons de la mise en œuvre de cette approche au sein du PANPF, et de partager les bonnes pratiques avec d'autres pays du Partenariat de Ouagadougou et au-delà.

La présente revue documentaire est la première phase de l'étude qui comprend aussi une recherche qualitative avec des acteurs de la PF et des femmes bénéficiaires La revue documentaire a pour objectifs de : i) évaluer et analyser la documentation existante et disponible ; ii) identifier les institutions et personnes jouant un rôle clé dans les réalisations du plan afin d'approfondir l'analyse de la mise en œuvre de l'approche 3D à travers les interviews planifiées dans la deuxième phase de l'étude ; iii) focaliser la documentation sur les aspects ayant jusqu'à présent reçu le moins d'attention. 


\section{Méthodologie}

Cette étude est menée en collaboration avec la DSRSE, l'USAID et le Partenariat de Ouagadougou. Plusieurs étapes ont été nécessaires à la conduite de cette revue documentaire :

- Inventaire de la documentation existante auprès des parties prenantes

Pendant la phase de développement du protocole de recherche de l'étude, une requête faite auprès du MSAS et d'autres acteurs pour recueillir les documents disponibles sur l'approche 3D et sa mise en œuvre a été peu concluante. Une fois l'étude approuvée et commencée, tous les acteurs impliqués dans au moins l'un des « $\mathrm{D}$ » de l'approche ont été contactés afin de les informer de l'étude et de recueillir toute documentation disponible sur les thématiques ou projets identifiés en lien avec l'approche 3D. Il s'agit notamment de parlementaires, représentants du gouvernement, autorités locales, gestionnaires de programmes, représentants d'organisations non-gouvernementales $(\mathrm{ONG})$ nationales et internationales et représentants des organisations de la société civile. Des rendez-vous ont été organisés pour, d'une part recueillir la documentation disponible à leur niveau, et d'autre part favoriser des échanges oraux pour fournir les explications demandées sur l'étude et collecter des informations complémentaires.

- Recherche de documentation en ligne

- Analyse de la documentation recueillie

Une lecture et une analyse approfondies des notes et documents obtenus ont permis d'extraire les éléments d'information sur la mise en œuvre de l'approche 3D.

Le présent rapport fait le point sur l'état des connaissances concernant la mise en œuvre de l'approche 3D au sein du PANPF. Chacune des composantes de l'approche a d'abord été analysée individuellement, puis l'approche 3D a été explorée dans sa globalité. 


\section{Démocratisation}

La « démocratisation » est conçue pour faciliter l'accès aux services par le biais d'une approche multisectorielle et participative.

\section{ACTEURS IMPLIQUES}

Sous l'impulsion du MSAS et de la DSRSE, de nombreuses initiatives de démocratisation de la PF ont été menées, impliquant de multiples acteurs gouvernementaux et non gouvernementaux, notamment les acteurs de la société civile, les ONG et les associations œuvrant aussi bien pour le plaidoyer pour la démocratisation de la $\mathrm{PF}$ que pour les actions concrètes portant sur l'offre de services.

La revue documentaire a permis de recenser plusieurs acteurs impliqués dans la démocratisation de la PF. Ainsi, on peut citer d'une part les structures gouvernementales telles que le MSAS (notamment la DSRSE, le Service National Education et Information pour la Santé (SNEIPS), et la Cellule de Santé Communautaire), et d'autre part la société civile et les PTF, dont:

- Le Projet Initiative Sénégalaise de Santé Urbaine (ISSU), qui est un projet déroulé sur cinq ans (20102014) et qui vise à soutenir le MSAS dans le cadre du repositionnement de la PF en vue de l'atteinte des OMD 4 et 5. L'objectif principal de ce projet est l'augmentation significative du TPC dans des zones urbaines défavorisées ciblées, en travaillant à l'augmentation de l'accès et de l'utilisation des services. Ce projet est coordonné par IntraHealth en partenariat avec des ONG internationales (Family Health International 360 (FHI360) et Marie Stopes International (MSI)), et des ONG et associations nationales (Enda Santé, Action et Développement (AcDev), ASBEF, Association Nationale des Sagesfemmes d'Etat (ANSFES), Agence pour le Développement du Marketing Social (ADEMAS), Réseau Islam et Population (RIP), le Réseau Siggil Jigeen (RSJ) et Réseau des Journalistes en Santé, Population et Développement).

- L’USAID à travers les agences d'exécution telles qu'Intrahealth, ADEMAS, le consortium sur la santé communautaire coordonné par ChildFund, Abt Associates....

- L'UNFPA

- L’Agence Japonaise de Coopération Internationale (JICA)

Des projets autres que ISSU interviennent pour la démocratisation de la PF, mais la documentation trouvée était insuffisante pour pouvoir les décrire ici.

\section{ENVIRONNEMENT POLITIQUE}

Depuis quelques années, on note un fort engagement du Gouvernement sénégalais dans le domaine de la SR. Le Sénégal s'est aussi fortement positionné pour la PF sur la scène internationale. Ainsi, en juin 2012, lors du Sommet de Londres sur la Planification Familiale, Madame la Ministre de la Santé a publiquement annoncé l'engagement du Sénégal à faire de la PF une priorité nationale en se fixant des objectifs ambitieux, notamment d'augmenter le TPC des 12\% reportés dans l'EDS 2010-2011 à 27\% en 2015 et de réduire les BNS d'environ 30\% à 15\% (Family Planning 2020a). En novembre de la même année, le PANPF a été lancé.

En 2012 la Division de la Santé de la Reproduction (DSR) a été érigée en Direction de la Santé de la Reproduction et de la Survie de l'Enfant (DSRSE), et la Division de la Planification Familiale (DPF) a été créée 
en son sein (Mané et al., 2012). Aussi, l'engagement a été pris d'allouer des ressources humaines et financières supplémentaires à la DSRSE afin d'assurer l'exécution et le monitoring du PANPF (Family Planning 2020b).

La facilitation de la mise en œuvre des stratégies du PANPF par la mise en application de mesures concrètes marque aussi l'engagement du Gouvernement pour la démocratisation de la PF. On peut citer par exemple l'adoption des initiatives pour l'offre de la PF à base communautaire, la formation des Infirmiers Chefs de Poste (ICP) sur les Méthodes de Longue Durée d'action (MLDA), la standardisation des prix des contraceptifs au niveau national et la mise en œuvre de stratégies avancées d'offre de services.

De plus, la révision des dispositions règlementaires, a été amorcée, notamment en ce qui concerne la délégation des tâches. Bien que cette initiative signe l'implication des autorités pour la dynamisation de l'offre de services $\mathrm{PF}$, la codification en reste incomplète.

L'initiation d'une Journée Nationale de la Planification Familiale a également marqué l'engagement du Sénégal dans le repositionnement de la PF. Cette journée organisée par la DPF, avec le soutien de l'USAID, IntraHealth et du projet ISSU, avait pour objet de mobiliser les leaders, les autorités publiques et les communautés autour de la PF à travers des campagnes de sensibilisation et de communication. Les éditions de 2008 et 2009 ont été célébrées à Dakar. En 2010 et 2011, la journée a été décentralisée, respectivement à Missira et Niakhar, afin d'engager les acteurs au niveau régional (Gueye, 2011). En 2013, le lancement de la Journée a été opéré à Dakar sans toutefois qu'aucune manifestation ne se soit tenue en région ${ }^{3}$. Depuis lors, aucune manifestation n'a eu lieu dans le cadre de cette initiative.

\section{HARMONISATION DES PRIX DES CONTRACEPTIFS FOURNIS PAR LE GOUVERNEMENT}

Rendre accessibles les produits contraceptifs à toutes les femmes sénégalaises qui en ont besoin contribue à la démocratisation. Afin de lever les barrières financières à cet accès, un arrêté ministériel datant du 15 janvier 2003 fixe le prix de cession des contraceptifs dans les structures publiques. La circulaire du 26 juillet 2010 sanctionne cet arrêté. L’application de la circulaire concerne le prix des pilules, injectables, implants, dispositifs intra-utérins (DIU), et colliers utilisés pour la méthode des jours fixes. Les condoms (masculins et féminins) sont distribués gratuitement (Ministère de la Santé et de la Prévention, 2010).

\section{AUGMENTATION DES RESSOURCES FINANCIERES POUR LA PF}

Lors du Sommet de Londres sur la PF en 2012, l'Etat, par le biais de Madame la Ministre de la Santé, s'est aussi engagé à accroitre l'allocation budgétaire annuelle à la SR de 2,5 à 5\%, à augmenter de 200\% l'allocation budgétaire pour les produits contraceptifs (de 100 à 300 millions de francs CFA), et à augmenter le budget pour la gestion du programme de PF de 100\% (Family Planning 2020a).

Selon RSJ, à travers le Projet Advance Family Planning (AFP) dont l'un des objectifs est de s'assurer de l'augmentation effective de ces contributions, au 15 avril 2015, les deux tiers de l'engagement promis pour l'allocation budgétaire aux produits contraceptifs ont été effectifs. Les 100 millions restants devraient être effectifs entre juillet et décembre 2015 (délai que la DSRSE justifie par les investissements dans le Plan Sénégal Emergent, la création de l'Agence de la Couverture Maladie Universelle, la redistribution des allocations du MSAS avec l'intégration de l'Action Sociale à son portefeuille et l'épidémie d'Ébola). Un document permettant la traçabilité des fonds engagés pour la PF devrait aussi être fourni par le Gouvernement (Réseau Siggil Jigeen, 2015c).

3 Information recueillie lors de conversations téléphoniques. Pas de publication trouvée pour la documenter. 


\section{ACCROISSEMENT DU LEADERSHIP DES FEMMES: PROGRAMME "BAJENU GOX "}

Cette initiative a été développée en vue d'atteindre les objectifs du PNDS, par le biais de l'utilisation du leadership des femmes. Le programme "Bajenu Goxt", adopté par le MSAS, a pour but de contribuer à la diminution de la mortalité maternelle, néonatale et infantile. Il vise à accroitre l'utilisation des services de santé par les femmes lors de la grossesse, de l'accouchement, du post-partum et pour les enfants de 0 à 5 ans. Initié par la DSRSE et soutenu par le programme Santé USAID/Santé communautaire, ce programme est une réponse communautaire qui repose sur le leadership des femmes. Le rôle de la bajenu gox est de promouvoir des comportements favorables à la santé de la mère, du nouveau-né et de l'enfant, de soutenir, conseiller, suivre et orienter les femmes, tout en favorisant l'acceptation de la stratégie par les belles-mères, les grands-mères, les pères et les conjoints. Dans ce cadre, l'une de ces missions est de « sensibiliser pour le recours à la PF ».

Lancé en janvier 2009 à Kolda, le programme a ensuite été étendu dans les 14 régions du pays. En septembre 2012, on dénombrait 7343 bajenu gox formées et suivies. Le programme a donc été initié avant la naissance de l'approche 3D et du PANPF mais ayant montré de bons résultats, il s'est consolidé à partir de 2012. Dans le cadre du PANPF 2012-2015, il était prévu le recrutement et la formation initiale de 6500 bajenu gox supplémentaires. L'état actuel d'exécution de ce programme n'a pu être documenté.

En tenant compte de la population totale du Sénégal, l'objectif est d'au moins une bajenu gox pour 1000 habitants en milieu urbain. En milieu rural, il est d'au moins une bajenu gox par village ou hameau. Malgré le développement et l'élargissement du programme, des gaps de couverture persistent (DSRSE, 2012). La documentation sur les gaps actuels n'a pu être obtenue.

\section{RENFORCEMENT DE L'ACCES GEOGRAPHIQUE AUX SERVICES}

Contribuant à la démocratisation de la PF, plusieurs initiatives ont permis l'amélioration de l'accès aux services par du personnel qualifié ou l'amélioration de l'accès aux différentes méthodes dans toutes les zones et notamment les zones rurales.

Un financement de l'USAID a permis la mise en place par IntraHealth d'un projet pilote, sur la période 20062011, d'offre de MLDA (implants et DIU) par les infirmiers. Ceci était jusque-là réservé aux médecins et sagesfemmes. Aujourd'hui ces MLDA sont disponibles au niveau des postes de santé de 12 des 14 régions (Matam et Tambacounda exclues) et sont délivrées le plus souvent par les infirmiers.

La stratégie des Sages-Femmes Itinérantes (SAFI) est mise en œuvre par le MSAS et la Cellule de Santé Communautaire depuis 2015 et bénéficie d'un financement de la Fondation Bill et Melinda Gates. Elle consiste à mettre à la disposition des ICP des sages-femmes, appelées aussi SAFI, qui seront en itinérance pendant environ $60 \%$ de leur temps et travailleront le reste du temps dans la structures de santé. Le besoin en SAFI est estimé à 800 sages-femmes pour 2017. La sélection des régions basée sur les taux de mortalité maternelle et néonatale, le TPC et la proportion d'accouchements assistés par du personnel qualifié a positionné les régions de Matam, Kédougou et Sédhiou comme prioritaires. La SAFI est placée sous la responsabilité de l'ICP et ses activités permettront d'atteindre les populations les plus reculées, d'une part au niveau des cases de santé, et d'autre part grâce à l'itinérance au niveau des sites communautaires et lieux de rassemblement public tels que les marchés hebdomadaires. La SAFI appuie également la supervision et l'encadrement des acteurs communautaires de santé (Cellule de Santé Communautaire, 2015).

4 Expression en langue ouolof se traduisant par «marraine de quartier». La Bajenu Gox est une femme reconnue comme leader par sa communauté 


\section{RENFORCEMENT DE L'ACCES DES ADOLESCENTS/JEUNES AUX SERVICES}

Selon l'Organisation Mondiale de la Santé (OMS), les adolescents sont les personnes appartenant à la tranche d'âge 10-19 ans et les jeunes celles de la tranche 15-24 ans. Ainsi, ces deux tranches se recoupent. Ensemble, elles forment le groupe des adolescents/jeunes entre 10 et 24 ans (Division de la Santé de la Reproduction des Adolescent(s)/Jeunes, 2014).

Au Sénégal, les adolescents/jeunes se caractérisent par leur poids démographique élevé : ils représentent 31,3\% de la population totale, dont 22,6\% d'adolescents (Division de la Santé de la Reproduction des Adolescent(s)/Jeunes, 2014). Aussi, leur potentiel à contribuer à la croissance économique du pays est important, notamment grâce au dividende démographique, qui est défini comme la croissance économique rapide résultant de la baisse de la mortalité et de la fécondité du pays, et de l'évolution de la pyramide des âges de la population caractérisée par un nombre maximum de jeunes adultes, et relativement peu d'enfants et de personnes âgées, ce qui crée une opportunité de croissance plus rapide. Dans ce contexte, investir pour la santé et le développement des adolescents/jeunes est primordial (Partners in Population and Development Africa Regional Office, 2015).

Selon l'EDS-C de 2014, les adolescentes, qui constituent 22\% des femmes en âge de procréer, contribuent pour $9 \%$ à la fécondité totale des femmes. De plus, 18\% des femmes de 15-19 ans ont déjà commencé leur vie féconde, cette proportion atteignant 32\% à 18 ans. Malgré un TPC en hausse (12,3\% pour les méthodes modernes chez les 15-19 ans et 12,2\% chez les 20-24 ans selon l'EDS-C 2014 contre respectivement 1,9\% et 6,0\% selon l'EDS-MICS de 2010-2011), en 2014 les BNS concernaient 21\% des 15-19 ans (dont 20,3\% pour espacer les naissances) et 26,4\% des 20-24 ans (dont 26,0\% pour espacer) (ANSD et ICF International, 2012 ; ANSD et ICF International, 2015).

L'importante vulnérabilité de ces groupes, la faible prise en charge de l'éducation sexuelle dans les programmes scolaires et l'importance des mariages précoces font de ces groupes d'âge une cible essentielle des programmes SR en général et PF en particulier. Les principaux défis relatifs à la santé sexuelle et reproductive des adolescents/jeunes portent sur l'accès à des services adaptés à leurs besoins et à une information de qualité, la promotion de l'égalité des sexes et de l'autonomisation des femmes, l'amélioration de l'environnement social, législatif et réglementaire et la promotion d'initiatives multisectorielles (Division de la Santé de la Reproduction des Adolescent(s)/Jeunes, 2014). Dans cette optique, différentes initiatives en faveur de l'amélioration de la Santé de la Reproduction des Adolescents et des Jeunes (SRAJ) ont été engagées.

Dans le cadre du Projet Promotion des Jeunes institué en 1992, et suite à la CIPD de 1994 qui a attiré l'attention des autorités sur les besoins spécifiques des adolescents/jeunes en SR, plusieurs initiatives ont vu le jour. On peut citer par exemple la mise en place en 1996, à l'initiative du ministère de la Jeunesse soutenu par l'UNFPA, des Centres Conseil Ado (CCA), espaces d'écoute, de counseling et d'accompagnement des adolescents. Aujourd'hui 15 CCA sont répartis dans 11 régions du pays. De 1999 à 2004, le Population Council a mené une recherche opérationnelle sur l'amélioration de la santé des Adolescents/jeunes. La production du curriculum « Grandir en Harmonie » en 2007 par la DSR, le Population Council et FHI a notamment servi de base à la plateforme «Click info ado » et a permis la formation de pairs-éducateurs. Le document de Stratégie Nationale en Santé des Adolescents/Jeunes élaboré en 2005, puis le Plan Stratégique de Santé Sexuelle et de la Reproduction des Adolescents/Jeunes au Sénégal 2014-2018 opérationnalisent les politiques établies dans ce domaine. Récemment, la formation des prestataires en SRAJ et l'adoption des Standards des Services de Santé Adaptés aux Adolescents/Jeunes ont été les actions clés pour la démocratisation des services SR/PF auprès des adolescents/jeunes. 


\section{Standards des Services de Santé Adaptés aux Adolescent(e)s/Jeunes}

Afin de pallier les difficultés liées à la SRAJ et alimenter le processus d'amélioration continue de la qualité des services de santé à offrir aux adolescents et aux jeunes, un document intitulé «Standards des Services de Santé Adaptés aux Adolescent(e)s/ Jeunes » a été élaboré en 2011. Il est destiné aux Equipes Cadre de Régions (ECR) et de Districts (ECD) et à d'autres parties prenantes telles que les CCA, l'Inspection médicale des Ecoles (IME) et le Groupe pour l'Etude et l'Enseignement de la Population (GEEP). Il définit cinq standards (Division de la Santé de la Reproduction des Adolescent(s)/Jeunes, 2014):

- Standard 1 : Au niveau du point de prestation de service (PPS), tout(e) adolescent(e) ou tout(e) jeune, quelles que soient les circonstances, a accès aux informations et aux conseils appropriés à son état de santé, son développement et ses droits.

- $\quad \underline{\text { Standard } 2}$ : Tout PPS est organisé pour offrir à tout(e) adolescent(e)/jeune des services de qualité adaptés à ses besoins.

- $\underline{\text { Standard } 3}$ : Tous les prestataires ont les connaissances, les compétences, et les attitudes positives (requises) pour offrir des services adaptés aux besoins des adolescent(e)s et des jeunes.

- Standard 4 : Les membres de la communauté y compris les adolescent(e)s/jeunes facilitent la mise en place et l'utilisation des services de santé par les adolescent(e)s/ jeunes.

- Standard 5 : Le système de gestion des services de santé prend en compte de façon appropriée les aspects liés à la SRAJ.

\section{Interventions en milieu scolaire}

En 1990, le programme Education à la Vie Familiale en Matière de Population (EVF/MP) marque l'apparition des questions de population dans les curricula de l'enseignement élémentaire (Division de la Santé de la Reproduction des Adolescent(s)/Jeunes, 2014).

A partir de 1994, une collaboration entre le Ministère de l'Education Nationale et le GEEP permet une extension de ce programme dans l'enseignement moyen et secondaire, intitulée "Promotion de l'Education à la Vie Familiale » et ciblant les élèves de 12 à 19 ans et les professeurs. Le programme sensibilise et renforce les connaissances sur les questions de grossesses précoces, d'infections sexuellement transmissibles, et d'hygiène et droits des adolescents en SR (GEEP, 2015 ; Division de la Santé de la Reproduction des Adolescent(s)/Jeunes, 2014).

Le GEEP a aussi mis en place dans les lycées et collèges (GEEP, 2015) :

- Des clubs d'Education à la Vie Familiale (EVF), et des espaces d'information, d'éducation, de communication et de responsabilisation des jeunes

- Une approche pédagogique active

- Des supports éducatifs actualisés destinés à l'enseignement de la population

\section{Interventions extrascolaires}

De nombreuses initiatives d'éducation, de sensibilisation et de communication en matière de SR destinées aux jeunes ont vu le jour ces dernières années. Certaines, peu pérennes, ont dû être arrêtées. D'autres, au contraire, ont pu perdurer et toucher des milliers de jeunes. On peut citer de manière non exhaustive :

- La mise en place d'espaces ados/jeunes

Le MSAS, par le biais de la DSRSE, a lancé la mise en place d' " espaces jeunes » dans les structures de santé (espaces d'accueil, d'information, d'écoute, de conseil, de sensibilisation). Les ados/jeunes peuvent y être reçus 
par des pairs qui leur dispensent les services et informations dont ils ont besoin (Division de la Santé de la Reproduction des Adolescent(s)/Jeunes, 2014). Cependant peu de documentation a pu être retrouvée sur ce projet.

- Elargissement de l'accès aux services pour les jeunes par:

- L'implantation de centres MSI, dont un espace SRAJ depuis 2014 au sein du centre médicosocial de l'Université Cheikh Anta Diop de Dakar, financé par l'USAID. Un « centre jeunes », financé par l'UNFPA, est aussi opérationnel à proximité de l'Université Gaston Berger de Saint Louis depuis 2013 (MSI, 2014).

- La mise en place d'une journée gratuite d'offre de services pour les jeunes le dernier samedi du mois (surtout pour les jeunes déscolarisés) dans le centre MSI de Dakar (MSI, 2014).

D'autres associations et ONG (comme l'ASBEF, RESOPODEV, Medicos del Mundo) mettent en place des opérations de promotion, de prévention, et d'offre de services de SRAJ, mais au vu du peu de documentation retrouvée, ces interventions ne pourront être décrites ici. De même, les initiatives favorisant l'accès aux informations en SR, par le biais des Technologies de l'Information et de la Communication (TIC) utilisées par exemple par RAES ou SIS Afrique, ou par le biais d'un numéro vert mis en place par le SNEIPS, ne seront pas documentées ici (Division de la Santé de la Reproduction des Adolescent(s)/Jeunes, 2014).

\section{RENFORCEMENT DE LA COMMUNICATION}

Suite au lancement du PANPF, un plan national de communication a été adopté en 2012, avec pour but de «catalyser la discussion sur la planification familiale au sein du couple et de la famille, et, casser l'a priori négatif et les fausses perceptions afin de rendre la planification familiale une norme socialement acceptable, soutenue de manière transparente et largement utilisée ». Trois axes de communication ont été retenus : renforcement de la communication sur les effets secondaires et les rumeurs, renforcement de la communication au sein du couple et vulgarisation de la position de l'Islam sur la PF. La communication à travers les médias et l'implication des leaders religieux occupent une place de choix dans cette stratégie. Les objectifs principaux du plan de communication, devant être atteints avant décembre 2014, sont de: i) diminuer de $50 \%$ à $20 \%$ la proportion d'hommes âgés de 25 à 55 ans qui estiment que les personnes qui utilisent des contraceptifs finissent par avoir des problèmes de santé ; ii) augmenter de $52 \%$ à $75 \%$ la proportion des hommes âgés de 25 à 55 ans qui déclarent avoir discuté de la PF avec leur conjointe ; iii) augmenter de $10 \%$ la proportion d'hommes âgés de 25 à 55 ans qui déclarent que l'Islam est favorable à l'utilisation des méthodes contraceptives modernes (Cabinet SIC, 2014).

Plusieurs activités ont été menées pour ce renforcement. On peut citer l'implication du Réseau des Journalistes en Santé, Population et Développement. En effet, la participation accrue des professionnels des médias (radios, télés, presse écrite, presse online, blogueurs) dans le traitement et la diffusion des informations dans le cadre du repositionnement de la PF a été favorisée, notamment, par des ateliers d'orientation. Les activités de plaidoyer du RSJ pour obtenir une prise de conscience et un engagement des décideurs politiques et des leaders nationaux et municipaux sur l'importance du soutien à la PF pour l'atteinte des objectifs de développement ont également contribué à la démocratisation de la PF. Les actions menées dans le cadre de la campagne de communication «Moytou nef » et de l'implication des leaders religieux, sont des piliers de la campagne nationale de communication.

\section{Campagne de communication « Moytou nef»}

La campagne de communication sur la PF, lancée en septembre 2013, a été conçue et mise en œuvre par le SNEIPS et la DSRSE en collaboration avec les partenaires clés dans le domaine de la PF, notamment l'USAID (à travers ADEMAS), l'UNFPA, et IntraHealth (à travers le projet ISSU). Au niveau opérationnel, différents 
acteurs ont été impliqués : les districts sanitaires (médecins chefs de district, coordonnateurs SR, Educateurs pour la Santé (EPS), sages-femmes, bajenu gox, relais, téléopérateurs du numéro vert), les agences de communication, l'association des professionnels de santé, les élus locaux, les acteurs communautaires, les religieux, les média nationaux, régionaux et communautaires, les artistes (comédiens, chanteurs) et les sportifs (lutteurs, promoteurs de lutte, randonneurs) (Cabinet SIC, 2014).

Le slogan «Moytou nef» appelle à éviter les naissances rapprochées pour l'épanouissement de la femme et de la famille. L'adhésion des hommes à la PF et l'augmentation du pourcentage d'hommes déclarant soutenir leur conjointe pour l'utilisation d'une méthode moderne sont des objectifs majeurs de la campagne.

La stratégie a focalisé les interventions sur l'utilisation des «mass média » avec la diffusion de spots, d'émissions à la radio et à la télévision, d'animations musicales, d'articles de presse écrite et de messages à travers les réseaux sociaux et les moyens de transport urbain, ainsi que sur des activités de sensibilisation menées par des associations et agents de santé communautaire (Projet ISSU, 2014a).

Selon les résultats d'une évaluation à mi-parcours conduite par ADEMAS et SEN-Ingénierie, la campagne de communication a montré de bons résultats. En effet, plus de $80 \%$ des répondants ont déclaré avoir vu ou entendu les messages de la campagne et avoir une meilleure connaissance de la PF. La campagne a aussi permis de susciter un besoin d'information sur la PF, notamment pour les 18-29 ans (46,7\%), de faire changer positivement de position sur la PF (cas pour 23,7\% des hommes de 25-55 ans par exemple), et de stimuler la pratique (par exemple pour plus de 17\% des 18-29 ans) (Cabinet SIC, 2014).

\section{Implication des leaders religieux}

Les religieux représentent une cible importante pour promouvoir l'utilisation de la PF et sont impliqués de plusieurs manières. D’une part, l'un des objectifs spécifiques du plan opérationnel de plaidoyer sur la PF est d'amener les religieux à être plus favorables à la PF et à contribuer davantage à son acceptation. A cet effet, des comités régionaux ont été mis en place et les religieux ont été appuyés dans l'élaboration et le suivi de leur plan de plaidoyer (DSRSE et MSAS, 2013). D'autre part, le plan de communication national implique les religieux pour favoriser la démocratisation de la PF. Par exemple, dans le cadre du projet ISSU, plusieurs approches les engagent. On peut citer les causeries entre un imam et un groupe de fidèles, les prêches lors desquelles les imams clarifient la position de l'Islam sur l'espacement des naissances, les conférences religieuses ou encore les visites de proximité de membres du projet auprès de guides religieux musulmans et chrétiens (Projet ISSU, 2014a).

\section{DEVELOPPEMENT DU PARTENARIAT PUBLIC-PRIVE}

Dans le but d'atteindre les OMD, le MSAS s'est fortement positionné en faveur d'un Partenariat Public-Privé (PPP) fort et pérenne afin de pallier la rareté des ressources dans le domaine de la santé. De plus, l'un des domaines d'intervention du PANPF comprend l'extension de l'offre dans le secteur privé avec comme objectif de recruter 100000 nouvelles utilisatrices. Pour ce faire, l'une des actions stratégiques proposées repose sur la création d'une structure dédiée à la gestion multisectorielle des PPP ou l'adaptation d'une structure existante (DSR et MSAS, 2012). Cette cellule serait logée au sein de la DSRSE, mais lors de cette revue il n'a pas été trouvé de documentation quant à la mise en place et l'état actuel de ce projet.

Cependant, l'environnement juridique et réglementaire actuel freine ce partenariat et peut empêcher dans certaines situations le secteur privé de jouer son rôle. Des discussions devront par exemple être menées en ce qui concerne les prix pratiqués dans ce secteur et un débat engagé sur les lois relatives à la possibilité pour les structures privées, telles que les cabinets médicaux ou les cliniques, de détenir un stock de produits contraceptifs. 
Toutefois, différentes initiatives de PPP dans le domaine de la PF peuvent être citées :

\section{Les franchises sociales}

Le PANPF préconise le renforcement des stratégies de franchises sociales avec l'objectif d'atteindre 200 franchises sociales mises en place d'ici 2015, notamment en en créant 60 en 2012, 100 en 2013, 150 en 2014 et 200 en 2015.

En 2015, le réseau de cliniques «Blue Star» de MSI comprend 50 PPS franchisés dans neuf districts de la région de Dakar. L'affiliation au réseau permet aux prestataires privés (sages-femmes et infirmiers) d'avoir accès et de délivrer, outre les méthodes largement distribuées telles que pilules et préservatifs, des MLDA (implants, DIU...) de qualité et à un prix abordable.

Pour les franchises sociales, le PPP se manifeste par le partage des données mensuelles avec les districts sanitaires où sont implantés les cabinets franchisés et par l'accès aux produits de PF à travers les comptoirs des districts ou l'Informed Push Model (IPM) (MSI, Février 2015).

\section{Les stratégies avancées mises en place par le projet ISSU dans le domaine de la PF}

Le projet ISSU a permis l'intégration de la PF à des services de proximité existants de trois de ses partenaires Enda Santé, MSI et l'ANSFES- chacun agissant selon ses spécificités. Les stratégies avancées ci-dessous sont le fruit d'une collaboration entre les organisations concernées, leurs prestataires et les districts sanitaires, permettant ainsi de faciliter l'accès aux services par les populations à faible revenu ou vivant dans des zones éloignées dans douze régions.

- Cliniques mobiles Enda Santé

Initialement dédiée au dépistage et à la prise en charge des infections sexuellement transmissibles auprès des travailleuses du sexe, les cliniques mobiles Enda Santé ont permis de proposer gratuitement des services PF à ce groupe et d'élargir l'initiative aux femmes vivant dans des zones d'accès difficile de la banlieue. Ces cliniques mobiles ont permis de recruter 911 nouvelles utilisatrices PF en 2012 et 3551 en 2013 (Projet ISSU, 2014a).

- Equipes mobiles de MSI

Les outreach de MSI permettent de proposer des services PF dans les zones mal desservies et difficiles d'accès, en passant par les PPS, les centres culturels, les mairies et les marchés hebdomadaires.

Les six équipes mobiles MSI (Dakar, Thiès, Tambacounda, Kaffrine, Diourbel et Louga), dont les activités visent essentiellement à traiter les gaps constatés en termes de MLDA au niveau des PPS, ont permis d'atteindre 24527 clientes PF en 2014 (MSI, Avril 2015). Notons ici que, en parallèle du projet ISSU, d'autres partenaires participent au financement de certaines de ces équipes mobiles : l'USAID (pour Diourbel et Kaffrine) et l'UNFPA.

\section{- Sages-femmes mobiles}

Cette stratégie de l'ANSFES permet un appui aux services PF des structures de santé. Les sages-femmes des antennes décentralisées de l'association proposent gratuitement ces services dans les structures sanitaires de la zone d'intervention du projet ISSU. Ces sages-femmes ont permis de recruter 3193 nouvelles clientes PF en 2012 et 3 387en 2013 (Projet ISSU, 2014a). 
D'autre part, les «MS ladies » de MSI sont des sages-femmes mobiles qui interviennent dans les zones défavorisées de la région de Dakar. Elles ont permis de toucher 1456 clientes PF en 2014 (MSI, Avril 2015).

\section{Le marketing social}

ADEMAS œuvre pour la promotion de la connaissance et de l'utilisation de méthodes contraceptives par des techniques de marketing social, à travers des campagnes d'illustration, de promotion, et de communication, et par le biais de caravanes. Plusieurs produits contraceptifs ont été distribués grâce à cette approche : préservatifs masculins $\left(\operatorname{Protec}^{\circledR}\right)$ et féminins $\left(\right.$ Fagaru $\left.^{\circledR}\right)$, pilule Securil ${ }^{\circledR}$, et plus récemment l'injectable Securil ${ }^{\circledR}$ Press, équivalent du Sayana ${ }^{\circledR}$ Press.

MSI utilise aussi de son côté des techniques de marketing social. Un agent travaille avec les acteurs communautaires (relais, bajenu gox) des districts sanitaires, en amont de l'offre de services afin de créer la demande (MSI, Avril 2015).

Le PANPF formule l'objectif de développer le marketing social sur les prochaines années en ce qui concerne les MLDA et notamment l'implant.

\section{Implication des entreprises du secteur privé dans l'offre de services PF}

Dans le cadre de la Composante Santé Maternelle, Néonatale et Infantile (SMNI)/PF/Paludisme du programme Santé USAID/Sénégal mis en œuvre par IntraHealth, et dans le but d'accroitre la prévalence contraceptive en offrant des services PF de qualité sur le lieu de travail, 25 entreprises des régions de Dakar et Saint Louis ont été enrôlées. Ainsi, un plaidoyer a été mené auprès des entreprises, des formations sur le paquet PF et les MLDA et des kits utilisés à leur effet ont été offerts, les rapports entre les entreprises et la Région médicale ou le District sanitaire de couverture ont été renforcés par le biais d'un " mémorandum d'entente » (améliorant la collaboration par la transmission des données, la supervision formative et l'approvisionnement des entreprises en produits PF) et les employés et populations ont été sensibilisés sur l'existence de ces services par des journées portes ouvertes de PF (Gueye, 2011).

\section{DEFIS RENCONTRES}

D’après la revue documentaire, plusieurs défis majeurs peuvent être notés.

La démocratisation de la PF se heurte parfois aux barrières socioculturelles et aux fausses rumeurs. On peut citer notamment le tabou autour de la sexualité des adolescents/jeunes, ce qui rend difficile la démocratisation de la PF à leur égard. De plus, des barrières d'ordre religieux limitent l'accès à la PF, d'autant que certains leaders religieux très influents y sont réfractaires et diffusent des messages hostiles à son utilisation.

Les stratégies avancées montrent des résultats très intéressants, notamment en ce qui concerne l'accessibilité géographique de la PF, mais il faut toutefois souligner qu'elles fonctionnent sur financement extérieur, ce qui interroge sur la pérennisation de telles activités.

Au niveau du secteur privé, malgré le développement de PPP, des défis restent à relever. Des discussions devront être menées et une évolution des cadres juridique et réglementaire envisagée.

\section{LEÇONS APPRISES}

La revue des différents projets et interventions, développés précédemment pour illustrer la démocratisation de la PF, permettent de tirer plusieurs leçons. 
L'engagement politique et financier du Sénégal est essentiel pour le repositionnement de la PF et le renforcement de l'accès aux services de PF. Cet engagement a fortement contribué à renforcer l'accès aux services de PF. Il s'est manifesté à travers le soutien politique au programme et la mise en place de diverses initiatives, notamment l'introduction de l'offre de services à base communautaire, les stratégies avancées, l'implication d'autres ministères (tels que la Jeunesse et l'Education nationale) et des religieux, et le développement du PPP. L'utilisation des médias et la communication de proximité constitue un élément central pour la promotion des services de PF et une opportunité pour l'atteinte des objectifs. En effet, les campagnes de communication et de sensibilisation (ex : campagne "Moytou nef») ont fait leurs preuves dans la génération de la demande. Elles ont montré des résultats probants qui doivent s’inscrire dans la durée.

L'amélioration et l'élargissement de l'offre, tant du point de vue de la gamme des méthodes contraceptives que de l'accès géographique ou financier, sont des conditions complémentaires et nécessaires à la génération de la demande.

Enfin, les différentes collaborations entre les secteurs public et privé et les interventions nées de ces partenariats permettent de renforcer le système de santé national et de combler les gaps existants dans l'offre de services. Ces stratégies devraient être développées le plus largement possible, tout en s'assurant de la pérennité de telles interventions.

\section{BONNES PRATIQUES A EXPLORER DAVANTAGE}

Les Pratiques à Haut Impact (PHI) sont des interventions identifiées par un groupe technique consultatif d'experts internationaux et approuvées par de nombreuses organisations. L'efficacité des PHI, basée sur les preuves, est jugée intéressante et leur passage à l'échelle est recommandé pour une optimisation des ressources et l'obtention de bons résultats des programmes de PF (Family Planning High Impact Practices, 2014). Concernant la démocratisation de la PF, plusieurs bonnes pratiques ressortent de cette revue documentaire, dont deux sont des PHI éprouvées dans la prestation de services, et d'autres participent à créer un environnement propice.

Dans le cadre de la prestation de services, la revue a démontré que le marketing social et les services mobiles de proximité sont des PHI éprouvées, c'est-à-dire que leur mise en œuvre est recommandée sur la base d'un niveau de preuves suffisant. De plus, différents modèles de stratégies avancées ont été testés et ont montré des résultats intéressants, d'une part en renforçant les compétences et la quantité des ressources humaines disponibles au niveau des structures de santé, et d'autre part en permettant d'atteindre des populations éloignées (Projet ISSU, 2014a ; Cellule de Santé Communautaire, 2015). Ces stratégies pourraient être mises à l'échelle dans l'ensemble des zones difficilement accessibles des 14 régions du pays.

En outre, la revue a relevé plusieurs PHI visant à créer un environnement propice et à améliorer les comportements et les résultats des programmes de PF. Tout d'abord, on peut citer les stratégies de communication qui, par le biais de différents canaux, permettent aux populations d'accéder plus facilement à l'information.

L'implication des hommes et des leaders religieux dans le processus de démocratisation de l'offre de services PF reste essentielle. Ces derniers représentent une cible importante des actions de communication. Pour de meilleurs résultats, les efforts dans ce sens devront être poursuivis et accrus.

L'engagement du Gouvernement et le financement des produits et des services représentent aussi des PHI, de même que la mise en place de politiques gouvernementales favorables à la PF. Afin de dynamiser les activités, une modification des cadres règlementaires est nécessaire. Comme cela a été amorcé pour la délégation des tâches, notamment à travers la distribution à base communautaire de contraceptifs injectables, des discussions 
devraient être menées, par exemple, sur les questions relatives à la prescription obligatoire pour les pilules, ou l'autorisation aux pharmaciens de fournir des services de PF.

Enfin, l'intégration de services PF adaptés aux adolescents à travers l'application des standards de services est une PHI qui devrait être consolidée. L'intégration effective de l'éducation sexuelle dans les curricula devra aussi être considérée. 


\section{Démédicalisation}

La « démédicalisation » vise à permettre à des agents non médicaux de fournir une gamme variée de services PF aux populations vulnérables (rurales, urbaines défavorisées, pauvres et jeunes). La distribution à base communautaire (DBC) et la délégation des tâches sont les deux axes principaux de cette démédicalisation.

\section{ACTEURS IMPLIQUES}

L'ONG ChildFund est très impliquée dans le volet santé communautaire de la PF au Sénégal et mène des actions et évaluations sur une grande partie des programmes qui seront décrits ici.

D’autres acteurs tels que MSI, Enda Santé, le projet ISSU, et l'ASBEF, à travers les stratégies avancées citées dans la partie «Démocratisation», participent aussi à la démédicalisation de la PF.

\section{DEVELOPPEMENT DE LA PF AU NIVEAU COMMUNAUTAIRE}

\section{Références dans les textes officiels}

La santé communautaire est un axe majeur du renforcement du système sanitaire au Sénégal, y compris dans le domaine de la PF. Les textes officiels y faisant référence sont décrits ci-dessous.

- L'arrêté ministériel Nº 07507 du 24 Août 2010 porte création et organisation du Programme «Bajenu Gox», composante essentielle de la santé maternelle communautaire.

- La promotion des initiatives à base communautaire fait aussi partie du Plan Stratégique de la Santé de la Reproduction 2011-2015 qui vise à accroitre le niveau d'utilisation des services de SR (DSR et MSAS, 2011).

- L'extension de l'offre à base communautaire est un domaine d'intervention à part entière dans le PANPF. Ainsi, à l'horizon 2015, le PANPF prévoit le recrutement de 125000 nouvelles utilisatrices PF au niveau communautaire, ce qui représente 36\% de l'objectif national (DSR et MSAS, 2012). Pour ce faire, diverses actions stratégiques sont menées, telles que l'élargissement de l'accès à la distribution par les cases avec des Agents de Santé Communautaires (ASC) lettrés et des ASC analphabètes, la revitalisation du programme «Bajenu Gox», l'utilisation des relais, l'accroissement de la couverture en Agents de Santé à Base Communautaire (ASBC), ou la distribution par d'autres catégories de Travailleurs Communautaires de Santé.

- Une cellule de santé communautaire, rattachée à la Direction Générale de la Santé du MSAS, et responsable de l'élaboration des stratégies de santé communautaire, de la coordination multisectorielle, de la promotion de la santé communautaire et du suivi et évaluation des activités a été créée en 2013 dans le cadre du Plan National Stratégique de Santé Communautaire 2014-2018. Ce plan a également prévu le renforcement de la couverture en cases de santé fonctionnelles et en sites communautaires, le lancement de la stratégie SAFI et d'un projet pilote sur les sages-femmes en réseau, ainsi que la garantie de la disponibilité des produits au niveau communautaire (Cellule de Santé Communautaire et MSAS, 2014).

\section{Projets exécutés avant et depuis la mise en ouvre de l'approche 3D}

Le programme de Santé de l'USAID/Santé Communautaire (PSSC) mis en œuvre par ChildFund porte sur différents volets de la santé communautaire en PF avec par exemple l'offre initiale de pilule (OIP) dans les cases de santé, l'offre initiale de contraceptifs injectables (OII) au niveau communautaire, y compris maintenant le Sayana ${ }^{\circledR}$ Press. La phase I du programme (2006-2011) visait à renforcer l'accès à un paquet de base de soins 
primaires de santé, y compris la $\mathrm{PF}$, dans les communautés rurales, au sein des cases de santé. Cette première phase a permis d'appuyer 1620 cases de santé dans 13 régions. Dans le domaine de la PF en particulier, il a été possible d'intégrer l'OIP à 470 cases de santé, de former 6069 ASC, matrones et relais à la promotion de la PF et à l'OIP, et de recruter 5733 utilisatrices de la PF au niveau des cases de santé (USAID Sénégal, 2011). Dans le cadre de la phase II du programme (2011-2016), ChildFund et ses partenaires (Africare, Catholic Relief Services, Plan International, Word Vision, Enda Sahel et Enda Santé) ciblent l'extension des services de santé communautaire aux 14 régions (Mbow, Ningue, Diop et al., 2015a).

Les agents de santé communautaires englobent les matrones, ASC, relais, bajenu gox, et ASBC. Ils se déplacent de porte à porte afin de fournir des informations sur la santé et de fournir des services de PF (collier du cycle, préservatifs, renouvellement de pilules). Ce programme, appuyé par l'UNFPA, est mis en place dans les régions de Matam, Saint-Louis, Tambacounda et Kédougou. Les ASBC y travaillent avec des ONG locales (Advancing Partners and Communities, 2013).

\section{DELEGATION DES TACHES}

\section{Références dans les textes officiels}

L'une des actions proposées par le PANPF concerne l'approfondissement de la délégation des tâches avec l'intégration de l'OIP dans le paquet intégré de services de toutes les cases de santé fonctionnelles (600 cases).

Parmi les documents officiels, la circulaire du 11 aout 2014 du MSAS autorise et promeut l'offre initiale de contraceptifs injectables sous forme intramusculaire (Depo-Provera ${ }^{\circledR}$ ) et sous-cutanée (Sayana ${ }^{\circledR}$ Press) au niveau communautaire par les matrones et ASC des cases dans tous les districts sanitaires (MSAS, 2014).

\section{Projets exécutés avant et depuis la mise en œuvre de l'approche 3D}

Depuis les années 1990, les autorités sénégalaises encouragent la DBC de produits PF. Parmi les premières initiatives, on peut citer:

- Le renforcement des capacités des matrones et ASC pour le counseling initial PF, effectif dans la grande majorité des structures de santé et permettant aux prestataires qualifiés de se focaliser sur des tâches plus spécifiques.

- L'OIP par les matrones, dont le projet pilote a été mené en 2008 par ChildFund sur financement USAID, a été étendu de huit régions à l'ensemble du pays.

A partir de 2012, avec la mise en œuvre du PANPF, la délégation des tâches a connu de réelles avancées, notamment avec l'offre des injectables par le personnel communautaire.

Les ONG ASBEF et AcDev ont mis en œuvre un programme d'offre de pilules et d'injectables par les relais communautaires, respectivement dans le district de Koumpentoum en 2012 et dans celui de Guédiawaye en 2013. Les clientes PF potentielles sont identifiées lors de causeries, mobilisations sociales, visites à domicile et niches. Elles bénéficient d'une visite à domicile, d'un counseling, et de la distribution de pilule s'il s'agit de la méthode choisie ou de l'orientation vers une structure de santé pour la prescription d'une autre méthode par une sage-femme. Les clientes bénéficient aussi d'un suivi régulier. Les résultats concluants de ce programme permettent d'envisager un passage à l'échelle et l'élargissement des méthodes proposées (Mbow et al. 2015a).

En 2012-2013, une initiative menée par ChildFund et incluant d'autres partenaires tels que FHI 360 et le Centre de Formation et de Recherche en Santé de la Reproduction (CEFOREP), a étudié la faisabilité de la dispensation du contraceptif injectable d'acétate dépôt médroxyprogestérone sous forme intramusculaire, DMPA-IM (Depo Provera $^{\circledR}$ ) par les matrones et ASC dans les cases de santé. Les résultats positifs de cette étude ont permis de 
confirmer la possibilité d'élargir la gamme de contraceptifs disponibles dans les cases de santé. Ainsi, sur la base de cette expérience communautaire réussie, le MSAS a ordonné, par le biais d'une lettre circulaire datée d'aout 2014 et adressée aux instances sanitaires régionales, le passage à l'échelle de cette offre d'injectable communautaire au niveau de tous les districts (Mbow et al., 2015a ; MSAS 2014).

En 2012, un projet collaboratif entre PATH, FHI 360 et le CEFOREP et soutenu par le MSAS, a permis l'introduction au Sénégal du DMPA sous forme sous-cutanée auto-injectable (Sayana ${ }^{\circledR}$ Press) et sa distribution à base communautaire par les matrones et ASC. Les résultats favorables de ce projet pilote incluant 12 cases de santé a abouti à l'élargissement de cette initiative à tout le pays, officialisé par la circulaire du MSAS en date du 11 aout 2014 (Mbow et al., 2015a).

\section{DEFIS RENCONTRES}

On constate parfois des difficultés de planification et de coordination des interventions sur le terrain qui se manifestent par le télescopage des activités dans les mêmes zones. Ces difficultés peuvent être résolues en améliorant la planification et la synergie entre les différents programmes (Projet ISSU, 2014a ; Mbow, Ningue, Diop et al. 2015b).

Des ruptures en intrants peuvent limiter le travail des agents communautaires dans la prestation des services. D'une part, l'IPM qui a permis de pallier en grande partie les ruptures de stocks en produits PF ne permet pas d'approvisionner les cases de santé qui sont toujours tributaires des postes de santé pour leur approvisionnement. D'autre part, les intrants nécessaires à l'administration de certaines méthodes (pansements, anesthésiques...) sont encore touchés par des ruptures de stock et un cout élevé (Projet ISSU, 2014a). Un système d'approvisionnement efficace permettant d'éviter les ruptures de stock doit être mis en place, et les initiatives amorcées dans ce sens doivent être passées à l'échelle.

La fidélisation des agents de santé dans les programmes de santé communautaire est nécessaire pour assurer la continuité des programmes. Or, la faible motivation de ces agents est souvent à l'origine de leur instabilité dans les programmes (Mbow et al. 2015b).

La question de la qualité des services des cases de santé, notamment liée à la capacité de supervision et de suivi des agents par l'ICP qui en est responsable, reste un défi à relever.

\section{LEÇONS APPRISES}

Cette revue documentaire a permis de tirer des leçons quant à la consolidation de l'approche de démédicalisation de l'offre de services PF.

Les activités de sensibilisation soutenues par les agents communautaires permettent d'accroître la demande et l'utilisation des services; de même l'implication des communautés rend ces activités pérennes. L'exemple des bajenu gox, dont le programme est fondé sur le patrimoine culturel sénégalais et le leadership des femmes, montre bien que cela contribue à une meilleure acceptation du message par les bénéficiaires.

Des agents de santé communautaires bien formés et bien supervisés sont capables d'offrir, outre les activités d'éducation et de sensibilisation, des services PF adéquats et notamment les injections de Depo Provera ${ }^{\circledR}$ en intramusculaire ou Sayana ${ }^{\circledR}$ Press en sous-cutané et les implants (USAID Sénégal, 2011 ; Mbow et al. 2015b). 


\section{BONNES PRATIQUES A EXPLORER DAVANTAGE}

Les agents de santé communautaires permettent d'atteindre des femmes rurales ayant une accessibilité difficile aux structures de santé. Aussi l'appui des ASC pour fournir une gamme plus large de méthodes de PF est considéré comme une PHI (Family Planning High Impact Practices, 2014). Il conviendra donc de développer la formation, l'équipement et le suivi des agents impliqués dans les activités réussies de transfert des tâches et d'offre de services à base communautaire pour contribuer à atteindre les objectifs fixés en matière de PF. 


\section{Décentralisation}

La « décentralisation » vise à consolider les systèmes de santé aux niveaux régional, district et communautaire, tout en renforçant la bonne gouvernance, la responsabilisation et la collaboration intersectorielle.

En 1996 les collectivités locales sénégalaises ont acquis une autonomie de gestion et plusieurs domaines de compétence leur ont été transférés de l'Etat, notamment les actions de développement sanitaire et social. En 2013, l'Acte III de la décentralisation a été adopté et mis en œuvre. Concernant la PF en particulier, depuis la naissance de l'approche 3D, la décentralisation est devenue un objectif à part entière. D'une part, la décentralisation se manifeste par la responsabilisation des différents niveaux de la pyramide sanitaire, et plusieurs initiatives pilotes sont aujourd'hui progressivement étendues à tous les niveaux et à toutes les zones. D'autre part, les collectivités locales ont le devoir de s'impliquer financièrement.

\section{ACTEURS IMPLIQUES}

Les acteurs impliqués dans la décentralisation englobent tous les niveaux de gestion de la pyramide sanitaire mais aussi les différentes organisations travaillant à la mise en œuvre de projets et à leur passage à l'échelle. On peut citer ChildFund en ce qui concerne les aspects de la santé communautaire sur le terrain, PATH, MSI, ASBEF, et ADEMAS pour l'introduction progressive du Sayana ${ }^{\circledR}$ Press et Securil ${ }^{\circledR}$ Press au Sénégal, et tous les acteurs impliqués dans le projet ISSU et son passage à l'échelle. La décentralisation fait aussi appel aux instances pharmaceutiques au niveau central, régional et du district.

\section{EXTENSION A TOUS LES NIVEAUX DE LA PYRAMIDE SANITAIRE ET TOUTES LES ZONES}

\section{Passage à l'échelle de l'OIP et de l'OII}

Le PANPF prévoit le passage à l'échelle de l'OIP. Dans un premier temps, il est prévu à terme l'inclusion de l'OIP dans le paquet intégré de services de toutes les 600 cases fonctionnelles avec d'abord la cartographie des cases qui ne sont pas encore passées à l'OIP et des cases éligibles (matrones ou ASC alphabétisés), puis la traduction des outils en langues locales, la formation à l'OIP des ASC et matrones alphabétisés, la dotation des nouvelles cases proposant l'OIP en stock initial de pilules, le suivi post formation et la supervision par l'ECD pendant dix mois. Dans un deuxième temps, le plan prévoit la poursuite de projets pilotes et l'évaluation de la faisabilité de l'extension de l'OIP aux matrones et ASC analphabètes. Enfin, dans un troisième temps, le plan prévoit le lancement d'études pilotes pour évaluer et valider la pertinence de l'extension de l'OIP aux autres travailleurs communautaires de santé (relais et bajenu gox) (DSR et MSAS, 2012).

L'OII intramusculaire suit le même processus de mise à l'échelle progressive, avec l'extension d'abord aux matrones et ASC alphabétisés dans les cases fonctionnelles, puis l'évaluation de la faisabilité de l'extension aux matrones et ASC analphabètes, puis aux relais et bajenu gox (DSR et MSAS, 2012).

\section{Introduction du Sayana ${ }^{\circledR}$ Press par différents canaux}

Parallèlement à l'extension de l'OIP et de l'OII, le Sayana ${ }^{\circledR}$ Press fait l'objet d'une étude pilote pour son introduction au Sénégal, coordonnée par PATH, en collaboration avec le MSAS, et impliquant divers partenaires tels que la Fondation Bill et Melinda Gates, l'USAID, l'UNFPA, le UK Department for International Development (DFID) et Pfizer. Cette étude s'établit sur la période 2013-2016 dans les régions de Dakar, Thiès, Saint-Louis et Fatick. Sayana ${ }^{\circledR}$ Press est distribué par différents canaux existants. D’une part, la méthode est offerte (simultanément au DMPA-IM) au niveau communautaire et au sein des structures 
sanitaires. Cette distribution est soutenue par l'UNFPA et le marketing social du produit par l'USAID. D'autre part, Sayana ${ }^{\circledR}$ Press est promu dans le secteur privé non lucratif par le biais du réseau de franchise Blue Star de MSI et du réseau de cliniques de l'ASBEF. Sayan $a^{\circledR}$ Press est également disponible dans les pharmacies privées, sous le nom de Securil ${ }^{\circledR}$ Press dont ADEMAS est chargé du marketing social.

ChildFund est responsable de la formation des prestataires au niveau communautaire et IntraHealth de celle des autres fournisseurs de prestation en Sayana ${ }^{\circledR}$ Press dans le secteur public et le secteur privé (Division de la Planification Familiale, 2013).

Une étude relative à la faisabilité de l'auto-injection du Sayana ${ }^{\circledR}$ Press avec les matrones dans les cases de santé a débuté en mi-2015 (PATH, 2015).

\section{Passage à l'échelle des initiatives du projet ISSU}

Le projet ISSU, avec l'objectif d'augmenter significativement le TPC dans les zones urbaines défavorisées, a ciblé Dakar, Pikine, Guédiawaye et Mbao lors de la première phase, puis Mbour et Kaolack ont été ajoutées lors de la deuxième phase. Le testing du « paquet porteur » à Rufisque et Diamniadio a également été mis en œuvre. Un ensemble d'approches a été modélisé autour de trois composantes :

- Composante offre : IPM, approche sage-femme (20 sages-femmes mises à disposition des PPS pour offrir des services PF), franchise sociale avec les PPS Blue Star de MSI, Identification Systématique des Besoins de la Cliente en PF (ISBC/PF), stratégies avancées matérialisées par des offres de proximité (cliniques mobiles, outreach, consultations foraines municipales gratuites), OIP en milieu urbain (OIPUB) dans les cases de santé, et approche qualité.

- Composante demande : plaidoyer religieux, activités communautaires (niches, conversations communautaires et visites à domicile), communications de masse et counseling, marketing et référence vers les PPS de proximité par les pharmacies pilotes.

- Composante plaidoyer : mise en place de comités locaux de plaidoyer et d'un Observatoire National de la Planification Familiale.

L'évaluation à mi-parcours du projet, exécutée par Measurement, Learning and Evaluation (MLE) en 2013, a montré des résultats très satisfaisants notamment du point de vue de l'augmentation du TPC entre 2011 et 2013, qui est encore plus visible pour les quintiles les plus pauvres : de $17,8 \%$ à $30,8 \%$ pour Guédiawaye, de $13,0 \%$ à $27,1 \%$ pour Pikine et de $11,1 \%$ à 20,5\% pour Mbao. De nettes améliorations ont également été notées du point de vue de la sécurisation des produits contraceptifs grâce à l'IPM (fin des ruptures de stock à Pikine et Guédiawaye en 2013) et de l'évolution de l'offre des MLDA (de 12,0\% à 28,7\% à Pikine, Guédiawaye et Mbao) (Projet ISSU, 2014b). 
Suite à ces résultats intéressants, en 2014, un processus de capitalisation des approches porteuses du projet a démarré, ayant pour principal enjeu le passage à l'échelle des initiatives réussies au Sénégal et dans les autres pays francophones de la sous-région. Le " paquet porteur » retenu suite au processus de capitalisation des approches est défini ci-dessous (Figure 1):

FIGURE 1 | PAQUET PORTEUR DU PROJET ISSU
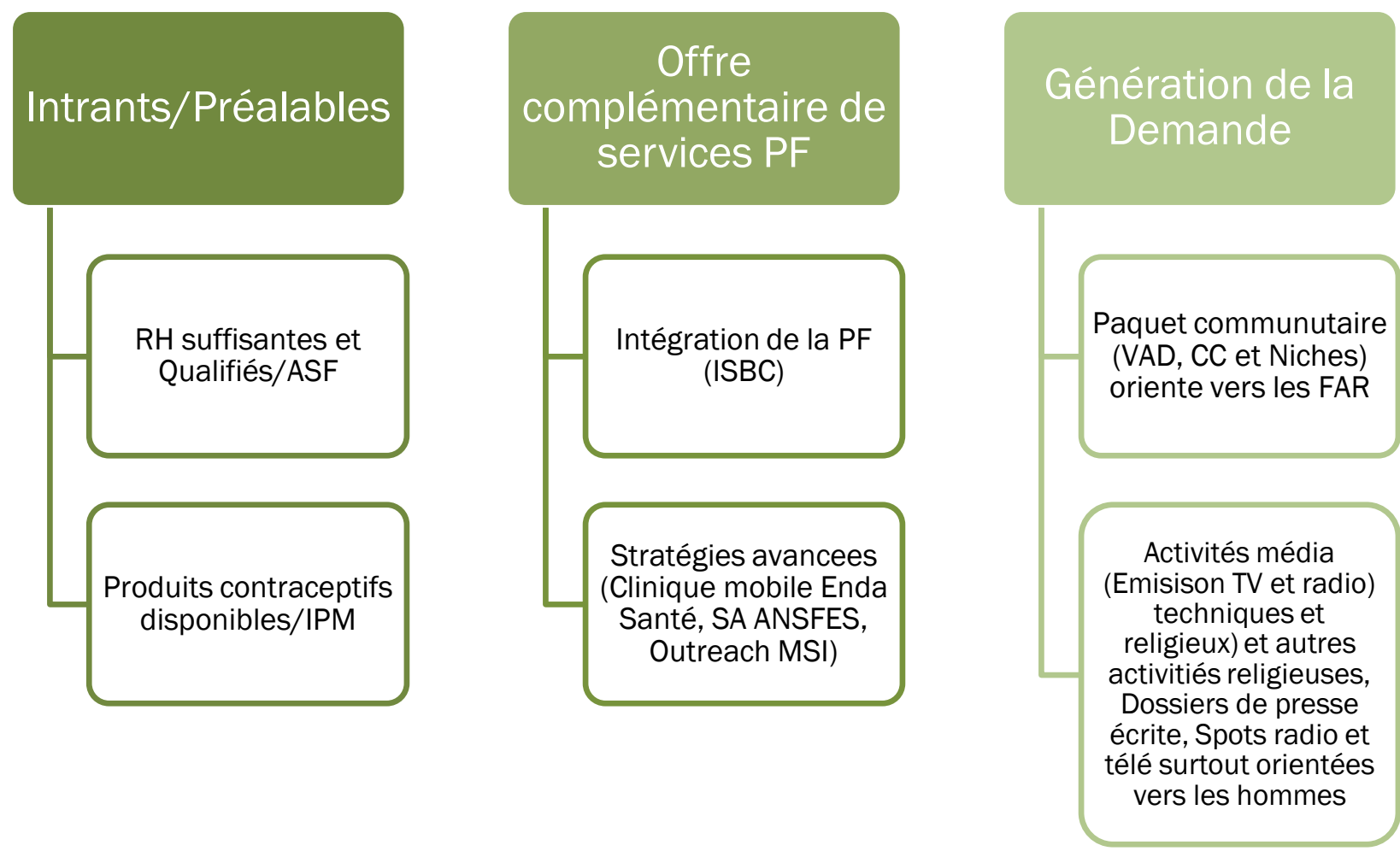

Source: Rapport de la capitalisation des approches du «paquet porteur» des interventions du projet ISSU. Novembre 2014

En 2014-2015, lors du testing du passage à l'échelle national, un "paquet porteur adapté » a été mis en place dans les districts sanitaires de Rufisque et Diamniadio. Il s'agit d'un paquet minimum adapté au contexte du district après identification des domaines nécessitant un renforcement. Après évaluation finale de MLE en fin 2015, la stratégie de diffusion du paquet porteur à plus grande échelle sera portée par l'Observatoire National de la PF (Projet ISSU, 2014a).

\section{Amélioration de la disponibilité des produits : IPM, PRA et « Jegesi naa »}

Une disponibilité médiocre des produits $\mathrm{PF}$, à l'origine de nombreuses ruptures stocks à différents niveaux de la pyramide sanitaire, a été analysée comme étant un frein majeur à l'offre de produits PF. Aussi, plusieurs initiatives sont nées tant au niveau de la Pharmacie Nationale d'Approvisionnement (PNA) avec les Pharmacie Régionale d'Approvisionnement (PRA) et PRA mobiles, que dans le cadre du projet ISSU avec l'IMP.

- Informed Push Model (IPM)

En 2010-2011, dans le cadre de l'enquête de base de ISSU, une étude de la chaîne d'approvisionnement de 33 établissements publics des districts de Pikine et Guédiawaye avait révélé des ruptures de stock très importantes des produits contraceptifs : à Pikine on notait 156 jours par an de rupture de stock pour l'injectable Depo Provera ${ }^{\circledR}$ et 300 jours par an pour l'implant Jadelle ${ }^{\circledR}$, soit respectivement $43 \%$ et $83 \%$ de l'année, avec au 
moins $60 \%$ des ruptures de stock survenues malgré la présence de stocks disponibles au niveau national. Le Gouvernement et ISSU ont alors développé un modèle de distribution de type "Push » (système de réception passive sans commande), auquel des opérateurs logistiques privés ont été associés. Ce système permet de simplifier le processus de commande, de limiter les intermédiaires et de rapprocher le point de la demande (les clientes des structures de santé) et celui de l'approvisionnement (le transporteur). Le système Push repose sur plusieurs points clés:

- Le stock initial de contraceptifs est fourni gratuitement à chaque centre de santé.

- Des logisticiens privés sont chargés du réapprovisionnement des centres mensuellement, afin de maintenir un niveau de stock minimum suffisant.

- Le centre ne paye que pour les produits qui ont été vendus et conserve la marge réalisée.

- Les logisticiens collectent les données de consommation de produits au moment de leur livraison et les renvoient au médecin chef du district.

- Les logisticiens sont payés sur la base de contrats à somme fixe. Durant l'étude-pilote, ces contrats ont été financés et gérés par le programme ISSU.

Le système de distribution en « Push » permet de simplifier les livraisons (Figure 2).

\section{FIGURE 2 | MODELE DE DISTRIBUTION EN "PUSH "}
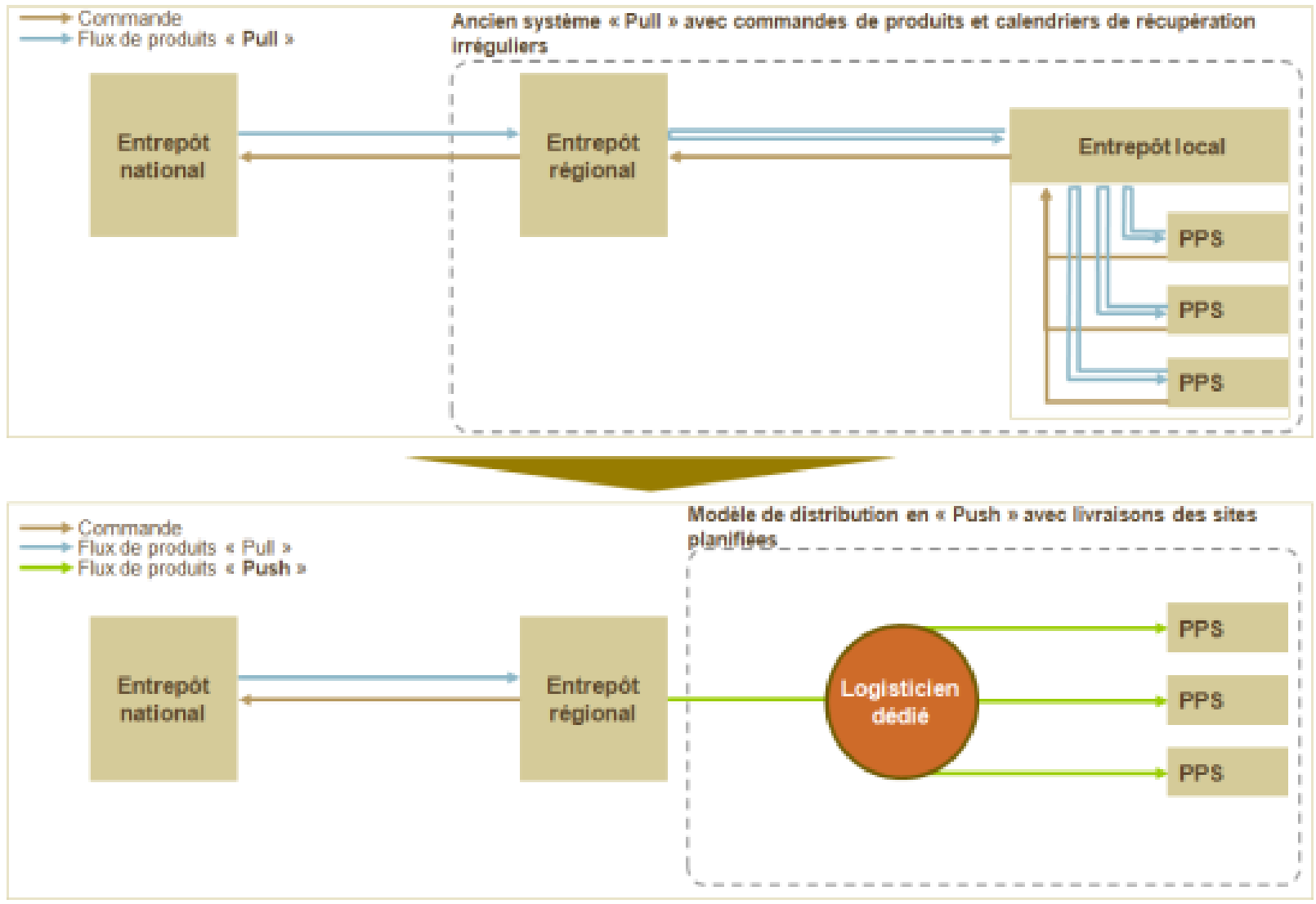

Source: Daffet al., 2014 
A partir de février 2012, le modèle a été testé pendant neuf mois dans 14 établissements publics du district de Pikine. Les ruptures de stock en pilules, injectables, implants et DIU ont complètement disparu à l'issu de cette phase pilote. Ce modèle a ensuite été étendu aux neuf districts de la région de Dakar au deuxième semestre 2012, puis à six régions en 2014, et à l'ensemble du territoire national en juillet 2015. Il a aussi été démontré que la disponibilité des produits et l'augmentation du TPC sont en étroite relation (Daff et al., 2014).

- Pharmacie Régionale d'Approvisionnement (PRA) mobile

Malgré un effort de décentralisation de la PNA dans le cadre de son programme de distribution de proximité, avec l'apparition des PRA dans 11 régions, les structures sanitaires des régions dépourvues de PRA (Sédhiou, Kaffrine et Kédougou) restaient enclavées par la distance qui les sépare de leur site d'approvisionnement et connaissaient encore d'importantes ruptures de stock. Le concept de PRA mobile a alors été développé en mars 2013. Il s'agit d'un modèle de distribution de proximité qui permet d'accéder à ces trois régions. L'approvisionnement des structures de santé de ces régions se fait par le biais d'un camion mobile, sans passer par l'interface des pharmacies régionales les plus proches. Ceci a permis d'améliorer l'accessibilité géographique des médicaments ainsi que l'accessibilité financière en évitant les coûts de transport jusque-là très élevés.

\section{- Projet « Jegesi naa »}

Le projet «Jegesi naa » a vu le jour dans le cadre de la seconde phase du programme de distribution de proximité de la PNA avec pour objectif de juguler les ruptures de stocks dans les zones périphériques. Il s'agit d'un modèle de distribution concertée qui permet d'étendre le réseau de distribution de la PNA jusqu'au niveau du district pour acheminer les médicaments directement aux dépôts des districts.

Comme pour l'IPM, le dépôt du district dispose gratuitement d'un stock initial de produits estimé sur la base des besoins des PPS. Ce stock est ensuite renouvelé mensuellement, sur la base d'un bon de commande, par livraison depuis la PRA (recevant elle-même son stock de la PNA). Les produits commandés sont alors enlevés par les PPS au niveau du dépôt de district. Le système permet aussi de faire remonter au niveau du district les données de consommation des PPS.

Ce système permet d'améliorer la disponibilité et l'accessibilité des produits au niveau des dépôts de districts et des PPS, notamment par l'augmentation des possibilités de commande des districts du fait de la levée de la contrainte des fonds et de celle des moyens de transport. De plus, le programme devrait permettre une meilleure collecte et remontée au niveau central des données de consommation et influer sur la mise en place d'un bon système de planification des approvisionnements au niveau régional, district et postes de santé (PNA, 2014).

\section{Tutorat Plus}

Le projet de renforcement de la prestation de services de santé du programme Santé de l'USAID, coordonné par IntraHealth, prévoit un volet de formation continue sur site à partir de l'approche Tutorat Plus. Cette méthode, qui vise l'amélioration des performances en fournissant une formation encadrée par un tuteur et basée sur les besoins spécifiquement identifiés des PPS, bénéficie en 2015 à plus de 1200 structures de santé publiques ou privées des 14 régions. Cette approche, tout en évitant aux prestataires de soins de s'absenter des structures de santé pour formation, permet un renforcement des compétences visant à améliorer les connaissances et les performances, ainsi que la gestion, l'efficacité et la qualité des services. L'augmentation de l'utilisation d'un Paquet Intégré de Services de Qualité (PISQ), l'amélioration de la gestion des ressources, des données, des services et des approvisionnements sont des points clés abordés par le Tutorat Plus. L'approche implique plusieurs parties prenantes : districts, PPS et communauté et permet de créer des partenariats pour l’amélioration de la qualité des services » (IntraHealth International, 2011; IntraHealth, 2012). 


\section{RESPONSABILISATION DES DIFFERENTS NIVEAUX DE LA PYRAMIDE SANITAIRE}

Le PANPF prévoit une approche nouvelle concernant la mise en œuvre des interventions de PF au Sénégal. En particulier, l'accent est mis sur la coordination et le suivi des activités PF avec une plus grande implication des régions. Ainsi, les objectifs du PANPF ont été définis en collaboration avec les régions et districts, et un TPC différencié par région a permis de fixer des objectifs en fonction du potentiel et des caractéristiques de chaque région. Ce potentiel prend en compte le poids démographique, le taux de BNS et des caractéristiques telles que le niveau d'urbanisation, la présence de zones périurbaines très peuplées, le nombre de femmes en âge de procréer ou encore la présence d'acteurs de terrain. Les régions ont été classées en " fort potentiel » (Dakar, Thiès, Diourbel), " potentiel moyen » (Saint-Louis, Kaolack, Fatick, Louga, Ziguinchor) et " potentiel faible » (Matam, Tambacounda, Kaffrine, Kolda, Sédhiou, Kédougou). Les objectifs d'augmentation du TPC varient de $60 \%$ à $270 \%$ selon les régions. D'importants efforts doivent être menés pour atteindre ces objectifs ambitieux. Au vu des premières évolutions des indicateurs (TPC national augmenté de $12 \%$ à 20\% entre 20102011 et 2014), ces objectifs pourraient potentiellement être atteints (DSR et MSAS, 2012).

Les stratégies d'actions sont aussi développées en collaboration avec les différents niveaux de la pyramide sanitaire, et notamment les ECR. La décentralisation implique également une supervision accrue des structures sanitaires par les Régions médicales et les Districts sanitaires.

Le renforcement des PPS est également un objectif de la décentralisation. Il s'agit, d'une part, de revitaliser le réseau de cases de santé, avec 226 cases qu'il était prévu de rendre fonctionnelles pour 2014 (2 140 l'étaient sur les 2366 existantes selon un recensement réalisé par ChildFund en 2013) et de construire et rendre fonctionnelles 145 cases à l'horizon 2018 ( 75 cases en 2016, 50 en 2017 et 20 en 2018). Il s'agit aussi de renforcer la couverture en sites communautaires avec un objectif de 11000 sites sur l'ensemble du territoire national (un site pour 500 habitants en zones urbaines et périurbaines). Un gap de 7817 sites a été évalué par ChildFund (3 183 sites actuellement fonctionnels, animés par 7435 relais) concernant essentiellement les régions de Dakar, St Louis, Louga et Kaolack, et il a été estimé que 7565 relais supplémentaires seraient nécessaires à l'animation de ces sites. Ainsi, l'objectif est d'augmenter progressivement le nombre de sites : 7500 sur cinq ans avec deux Acteurs de Prévention et de Promotion par site, dont 1500 sites pour Prestataires de Santé Communautaire (Cellule de Santé Communautaire et MSAS 2014).

Le lancement du projet SAFI, permet de renforcer l'offre de services PF au niveau des cases et des postes de santé ne disposant pas de sage-femme fixe. Les sages-femmes itinérantes seront aussi responsables des stratégies avancées notamment sur les marchés. Il a été estimé que chaque sage-femme devrait couvrir quatre à six structures. Le besoin de recrutement atteint environ 800 sages-femmes itinérantes à l'horizon 2017 (Cellule de Santé Communautaire et MSAS 2014).

Enfin, le renforcement des capacités des prestataires du secteur public, mené par IntraHealth dans le cadre de la composante SMNI/PF/Paludisme du programme Santé USAID/Sénégal, et plus particulièrement de l'accroissement de l'accès à des services PF de qualité, a permis des activités de formation dans 11 régions. En effet, prestataires et membres des ECR et ECD ont pu bénéficier de formations en counseling et en technologie contraceptive, notamment sur les MLDA, ce qui a permis la décentralisation de ces méthodes pour les rendre plus accessibles aux populations. De plus, les membres des ECR et ECD ont pu montrer un engagement plus fort pour la gestion des programmes PF (Gueye, 2011). 


\section{DECENTRALISATION DES FINANCEMENTS: IMPLICATION DES COLLECTIVITES LOCALES}

La décentralisation du système de santé comprend la décentralisation des financements, d'une part par le biais des fonds de dotation octroyés directement aux régions en remplacement d'une gestion jusque-là centralisée, et d'autre part l'implication des collectivités locales. En pratique, la participation financière des collectivités est compliquée par plusieurs facteurs comme par exemple la difficulté de prioriser la PF parmi les autres potentiels investissements pour des communes dont les moyens sont limités, et les possibles alternances politiques qui peuvent remettre en cause les engagements pris.

Le PANPF prévoit une section dédiée au plaidoyer pour l'augmentation du financement public pour la PF, notamment auprès d'élus locaux, comités de santé et collectivités locales. De plus, l'un des objectifs spécifiques du plan opérationnel de plaidoyer est d'obtenir l'engagement des collectivités locales à soutenir la PF, avec pour résultats l'augmentation de la part des fonds de dotation octroyée aux structures de santé, le recrutement de personnel technique qualifié et l'appui au renforcement des capacités des prestataires, ASC et matrones en PF.

Les journées de consultations foraines du projet ISSU ont ainsi permis d'engager les élus locaux dans le cofinancement des activités PF. De son côté, dans le cadre du projet AFP, RSJ mène aussi des actions de plaidoyer à Dakar, Saint Louis, Kolda, Kaolack, Louga, et Thiès. Le plaidoyer aborde la mobilisation de ressources à allouer à la $\mathrm{SR} / \mathrm{PF}$ dans le prochain budget des collectivités locales, la réhabilitation de structures sanitaires, le recrutement et l'affectation de personnel de santé et une meilleure implication des élus dans les activités de sensibilisation sur la PF (Gueye, 2011). Ainsi, en septembre 2014, le RSJ a pu notamment amener le maire de la commune de Pikine Djidah Thiaroye Kao (PDTK) à s'engager à octroyer un million de FCFA au district sanitaire de Pikine pour l'achat de produits contraceptifs. De la même manière, le maire de Mbao s'est engagé le 16 mai 2014 à accorder un million de FCFA en appui au programme PF de sa commune, et le 5 janvier 2015, le maire de Pikine Nord s'est engagé à allouer une subvention annuelle de dix millions de FCFA pour l'achat de produits contraceptifs de deux postes de santé de la commune. Des activités de plaidoyer sont également menées auprès des conseils municipaux des communes de Dalifort, Guédiawaye, Yeumbeul Sud, Sahm notaire, Diarème Limamoulaye, Derklé Dieuppeul et Tivaouane Diacksao (ces deux derniers s'étant engagés par écrit sans montant établi à la date de la signature), afin de les amener à s'engager à allouer une partie des fonds de leur budget municipal à la PF (Réseau Siggil Jigeen 2015 a, b, c). A ce stade, la documentation fournie ne nous permet pas de vérifier le suivi de ces engagements et de conclure à la mobilisation effective des fonds pour le financement décentralisé de la PF.

\section{DEFIS RENCONTRES}

Les principaux défis liés à la décentralisation portent d'une part sur le management décentralisé, incluant notamment la gestion du système d'approvisionnement et la capacité de management des ECR et ECD, et d'autre part sur le financement du programme PF au niveau régional, et local par les collectivités. De plus, le renforcement du partenariat entre les collectivités locales et les districts sanitaires est fondamental pour assurer une meilleure coordination des activités de santé, notamment de santé communautaire, et une supervision efficace par le personnel qualifié.

Dans le modèle de l'IPM, un retard dans le recouvrement des coûts des produits vendus peut entraîner un arrêt de livraison de la part du fournisseur privé (Projet ISSU, 2014a). L'intégration du système informatique Chanel à la gestion de données de l'IPM pour la gestion des stocks et des recouvrements permettra de pallier les difficultés que rencontrent certaines régions. 
Au Sénégal, les informations relatives à la PF sont le plus souvent basées sur les données issues du secteur public et il est difficile pour le niveau central d'obtenir des données complètes du secteur privé. Des solutions devront être trouvées afin de pouvoir intégrer ces données et obtenir des chiffres globaux.

\section{LEÇONS APPRISES}

La responsabilisation et l'implication des ECR dans la définition des objectifs a permis une meilleure appropriation du PANPF et de ses objectifs.

La disponibilité en ressources humaines qualifiées et en quantité suffisante à chaque niveau de la pyramide sanitaire est une condition nécessaire à la réussite des programmes PF et à l'atteinte des objectifs. Ainsi, le renforcement des PPS et l'appui du passage à l'échelle des interventions visant à augmenter la disponibilité en ressources humaines telles que la DBC et la délégation des tâches, sont des axes essentiels à l'amélioration de la couverture nationale en services PF et à l'atteinte des objectifs.

Des décideurs sensibilisés de façon appropriée et convaincus des avantages que procurent les investissements dans la PF pour l'amélioration des indicateurs sanitaires et économiques, peuvent s'engager à allouer des fonds. De plus, le plaidoyer auprès des décideurs pour la mobilisation de ressources humaines, par le recrutement de personnel qualifié en PF, est essentiel.

\section{BONNES PRATIQUES A EXPLORER DAVANTAGE}

La mise en place de nouvelles approches pour l'approvisionnement en produits PF et leur décentralisation a permis de limiter, voire éliminer, les ruptures de stock. Mettre en place une gestion efficace de la chaîne d'approvisionnement est par ailleurs une PHI et ces initiatives devront être évaluées rigoureusement et rapidement passées à l'échelle, si confirmées efficaces, afin que toutes les régions puissent bénéficier de leurs avantages (Family Planning High Impact Practices, 2014).

Soutenir le financement des produits et services de PF aux niveaux national et communautaire est aussi une PHI (Family Planning High Impact Practices, 2014). Le plaidoyer pour l'augmentation du financement public de la PF montre des résultats intéressants et devra être accentué. De plus, pour de meilleurs résultats, les régions et districts devront s'engager dans le plaidoyer, notamment en identifiant et recrutant les champions aptes à faire la promotion de la PF (DSR et MSAS, 2012). 


\section{L'Approche 3D dans sa globalité}

\section{ANALYSE DE LA MISE EN GUVRE DE CHAQUE COMPOSANTE}

Malgré des barrières socioculturelles persistantes limitant encore l'accès à la PF au Sénégal, la démocratisation des services et produits PF a connu de franches améliorations ces dernières années. La communication en matière de PF n'est plus taboue et les sources d'information ont pu se multiplier. L'accès aux services a aussi été amélioré tant au niveau financier que géographique.

La démédicalisation de la PF, à travers la délégation des tâches, bien que s'étant développée depuis les années 1990, a récemment connu un tournant avec la possibilité pour le personnel communautaire d'administrer les contraceptifs injectables. Le passage à l'échelle des différentes initiatives menées et l'intégration de nouveaux projets (concernant par exemple les pharmaciens) représentent les prochaines étapes afin de parvenir à une démédicalisation optimale pour la prestation de services PF.

La décentralisation a elle aussi été initiée depuis de nombreuses années dans le système de santé, mais des avancées importantes avec la mise en place de projets porteurs, tels que le projet ISSU ou l'introduction du Sayana ${ }^{\circledR}$ Press, ont eu lieu récemment. Cependant, des efforts supplémentaires doivent être menés pour la décentralisation des financements et le passage à l'échelle de la décentralisation de l'approvisionnement en produits PF à travers les programmes de la PNA, dont la pérennisation constitue un élément essentiel.

Les trois composantes de l'approche 3D restent très liées, imbriquées les unes aux autres et sont parfois difficiles à discerner au sein d'un projet. Il n'est donc pas aisé de juger de leur progression individuelle.

\section{AVANCEES DANS LE DOMAINE DE LA PF DEPUIS LA MISE EN CEUVRE DE L'APPROCHE 3D}

Le Sénégal a connu des avancées notoires dans le domaine de la PF au cours de ces cinq dernières années et on constate une prise de conscience accélérée de l'importance de la PF dans le développement sanitaire et économique du pays, notamment grâce à la corrélation avec le dividende démographique. Même si ces avancées sont difficilement imputables à une stratégie spécifique, le progrès noté coïncide avec la mise en œuvre de l'approche 3D.

Malgré certaines lacunes, telles que la dépendance vis-à-vis des partenaires extérieurs pour l'approvisionnement du pays en produits contraceptifs, l'implication politique et financière du Gouvernement est à la base des avancées perçues. Cette implication s'est concrétisée par l'engagement de Madame la Ministre de la Santé, notamment lors du sommet de Londres sur la Planification Familiale en 2012, tant en matière d'objectifs à atteindre que de promesses de financement de la PF. Le PANPF 2012-2015 est le fruit de cet engagement.

Suite aux efforts menés dans le cadre de la démocratisation, démédicalisation et décentralisation des services PF, le Sénégal a vu une nette amélioration du TPC entre 2010-2011 et 2014.

\section{CONTRAINTES A LA MISE EN GUVRE DE L'APPROCHE 3D}

L'une des principales contraintes à la mise en œuvre de l'approche 3D en général, et du PANPF en particulier, concerne les mécanismes de financement. D’une part, la contribution des décideurs à tous les niveaux, et notamment des autorités locales dont l'augmentation a été suscitée par le procédé de décentralisation, est nécessaire à l'appui des programmes PF locaux mais encore en deçà des investissements attendus. D'autre part, 
la question de l'importance de la participation des bailleurs internationaux dans le financement des programmes et activités de PF au Sénégal interroge sur deux points : la cohérence entre les promesses et les financements effectifs, et la pérennité des actions entreprises. Aussi une meilleure appropriation des questions relatives à la PF par les autorités nationales et locales, et la recherche de financements innovants pourraient pallier un financement dégressif des partenaires. Concernant particulièrement la fourniture des produits contraceptifs, l'approche globale du marché, en incitant l'investissement de façon coordonnée du secteur public, des ONG, du marketing social et du secteur commercial, permet d'augmenter la disponibilité des produits (HIP, 2014). L'engagement financier du Gouvernement, notamment avec la promesse d'augmenter l'allocation budgétaire aux produits contraceptifs, semble pour l'instant être tenu aux deux tiers (Réseau Siggil Jigeen, 2015c).

Une autre contrainte concerne l'organisation et la cohésion des acteurs dans le domaine de la PF. Malgré une volonté politique de coordination des acteurs et de leurs actions autour du PANPF, quelques incohérences et un manque de synergie entre les interventions des différents acteurs sont parfois notés. On peut citer par exemple des doublons d'activités de partenaires dans une même région ou la multiplicité d'acteurs communautaires sur une même zone qui peut être à l'origine d'une confusion des populations quant à leurs rôles. Cependant il faut souligner que le Comité Technique PF, regroupant la DSRSE et les PTF, joue un rôle de coordination des actions stratégiques qui tend à limiter cette contrainte.

Enfin, des barrières relatives à la législation en vigueur dans le domaine de la PF sont parfois un frein à l'évolution de la prestation de services. Aussi le cadre législatif et règlementaire requiert des réformes comme cela a été le cas avec la circulaire ministérielle permettant l'offre de contraceptifs injectables par les matrones et ASC. 


\section{Limites de la revue documentaire}

Cette revue documentaire sur l'approche $3 \mathrm{D}$ a fait face à une principale limite représentée par l'accès à la documentation. En effet, peu de documents ont pu effectivement être obtenus, même pour des activités et concepts bien connus. Ceci est dû à l'inexistence de document ou à la résistance ou non-disponibilité des parties prenantes les possédant. Il a été possible de balayer les principales actions menées pour démocratiser, démédicaliser et décentraliser la PF au Sénégal. Cependant les acteurs impliqués et les activités reportées n'en constituent pas le répertoire exhaustif.

Il a également été difficile de s'assurer que les promesses, notamment concernant les financements, sont tenues, et à quel degré les initiatives décrites dans les divers documents sont mises en œuvre sur le terrain. 


\section{Conclusion}

Née d'une volonté de changement de politique afin notamment de pallier les freins à l'accès à la PF, les difficultés relatives aux ressources humaines et le défaut de valorisation des actions menées au niveau communautaire, l'approche 3D a été mentionnée pour la première fois en Afrique de l'Ouest francophone lors de la Conférence du Partenariat de Ouagadougou en 2011 par le Directeur de la Santé de la Reproduction du Sénégal. Cependant, par la suite, hormis dans l'avant-propos du PANPF 2012-2015 de Madame la Ministre de la Santé, cette approche 3D n'est citée ou documentée nulle part.

Les bons résultats qu'a connus le Sénégal en matière d'évolution des indicateurs PF ces dernières années sont difficilement attribuables de façon directe à l'approche 3D en tant que telle, puisqu'elle reste jusqu'ici très peu décrite. Des efforts importants doivent être faits pour documenter au fur et à mesure et par toutes les parties prenantes les initiatives et expériences, pour améliorer le partage et l'utilisation au niveau national, régional et international des leçons apprises et bonnes pratiques. Toutefois, il parait évident que les différentes composantes de l'approche et les diverses activités contribuant à la démocratisation, démédicalisation et décentralisation de la PF forment un ensemble ayant contribué à l'évolution positive de la PF ces cinq dernières années.

L'un des aspects importants à mettre en avant dans les prochaines années en ce qui concerne l'application de l'approche 3D est le renforcement de l'approche multisectorielle et le mécanisme de coordination qui lui est inhérent. Les différents acteurs intervenant dans le domaine de la PF regroupent le secteur public (MSAS, et autres départements ministériels tels que le Ministère de l'Economie et des Finances, le Ministère de la Femme, de la Famille et de l'Enfance, le Ministère de l'Education Nationale et le Ministère de la Jeunesse), la société civile, les organisations à base communautaire, le secteur privé à but lucratif, les professionnels de la santé, les collectivités locales, et les PTF. Il s'agira pour l'ensemble de ces acteurs de mener des actions concertées et coordonnées pour faciliter l'atteinte des objectifs en PF.

La disponibilité des produits PF est une condition nécessaire mais non suffisante pour atteindre les objectifs de TPC. L'offre de services doit intégrer les activités de délégation des tâches et s'accompagner du renforcement des capacités des prestataires et de la création de la demande. Le passage à l'échelle des initiatives ayant montré des résultats probants devra rapidement être mis en œuvre.

De plus, le prochain plan et les objectifs en matière de PF devront porter une attention spéciale aux cibles particulières. D'une part, afin de lever le maximum de barrières socioculturelles qui freinent l'utilisation de la $\mathrm{PF}$, les efforts menés auprès des religieux et des hommes pour aboutir à leur implication et adhésion doivent être poursuivis. D’autre part, les adolescents/jeunes restent une cible importante.

Dans le contexte actuel où toutes les opportunités liées au dividende démographique doivent être exploitées, la promotion de la baisse de la fécondité et la mise en œuvre de politiques adéquates relatives à la PF sont essentielles. Des actions en faveur de l'accès à une information de qualité et adaptée aux besoins des populations, notamment des jeunes, de la connaissance de l'existence des services PF et de la possibilité d'y accéder sont primordiales.

La contribution de l'approche 3D doit être très clairement décrite et évaluée dans le prochain PANPF. Cela représentera un grand intérêt pour le domaine de la santé, et en particulier de la SR/PF, mais au-delà d'autres politiques nationales pourraient s'en inspirer pour participer au développement du Sénégal. 


\section{Références bibliographiques}

Advancing Partners \& Communities. 2013. Profil de Pays : Programmes de santé communautaire du Sénégal. Arlington, VA: Advancing Partners \& Communities.

Agence Nationale de la Statistique et de la Démographie (ANSD) [Sénégal], et ICF International. 2012. Enquête Démographique et de Santé à Indicateurs Multiples au Sénégal (EDS-MICS) 2010-2011. Calverton, Maryland, USA: ANSD et ICF International.

Agence Nationale de la Statistique et de la Démographie (ANSD) [Sénégal], et ICF International. 2013. Enquête Démographique et de Santé Continue (EDS-Continue 2012-2013). Calverton, Maryland, USA: ANSD et ICF International.

Agence Nationale de la Statistique et de la Démographie (ANSD) [Sénégal], et ICF International. 2015. Sénégal : Enquête Démographique et de Santé Continue (EDS-Continue 2014). Rockville, Maryland, USA : ANSD et ICF International.

Cabinet Sen Ingénierie Consult. Décembre 2014. Evaluation à mi-parcours de la campagne de communication sur la Planification Familiale 2013-2014. Rapport final. Dakar : Ministère de la Santé et de l'Action Sociale.

Cellule de Santé Communautaire et Ministère de la Santé et de l'Action Sociale. 2014. Plan National Stratégique Santé communautaire 2014-2018. République du Sénégal.

Cellule de Santé Communautaire et Ministère de la Santé et de l'Action Sociale. 2015. Document cadre de la stratégie des sages-femmes itinérantes. Dakar : Ministère de la Santé et de l’Action Sociale.

Daff BM, Seck C, Belkhayat H, Sutton P. 2014. Le système de distribution en «Push » des contraceptifs mis en place au Sénégal réduit les ruptures de stock et améliore la qualité des services de planning familial. Glob Health Sci Pact. 2014 ; 2(2):245-252. Disponible sur http://dx.doi.org/10.9745/GHSP-D-13$\underline{00171 .}$

Direction de la Santé de la Reproduction et de la Survie de l'Enfant. 2012. Rapport de la revue du Programme Bajenu Gox. Dakar : DSRSE.

Direction de la Santé de la Reproduction et de la Survie de l'Enfant (DSRSE) et Ministère de la Santé et de l'Action Sociale (MSAS). 2013. Plan opérationnel de plaidoyer sur la planification familiale 2013-2015. Dakar : DSRSE.

Division de la Planification Familiale, Direction de la Santé de la Reproduction et de la Survie de L'Enfant et Ministère de la santé et de l'action sociale. 2013. Plan d'introduction et d'évaluation de Sayana ${ }^{\circledR}$ Press au Sénégal. Dakar : DSRSE.

Division de la Santé de la Reproduction (DSR) et Ministère de la Santé et de l'Action Sociale (MSAS). 2011. Plan Stratégique de la Santé de la Reproduction du Sénégal 2012-2015. Dakar : MSAS.

Division de la Santé de la Reproduction (DSR) et Ministère de la Santé et de l'Action Sociale (MSAS). 2012. Plan d'Action National de Planification Familiale 2012-2015. République du Sénégal. Dakar : MSAS. 
Division de la Santé de la Reproduction des Adolescent(e)s/Jeunes, Direction de la Santé de la Reproduction et de la Survie de L'Enfant (DSRSE) et Ministère de la santé et de l'action sociale et (MSAS). 2014. Plan Stratégique de Santé sexuelle et de Reproduction des Adolescent(s)/Jeunes au Sénégal 2014-2018. Dakar : MSAS.

Gouvernement du Sénégal. 2005. Loi no 2005-18 du 5 août 2005 relative à la santé de la reproduction. Dakar: Gouvernement du Sénégal.

Gueye EB. 2011. Programme Santé USAID/Sénégal Composante SMNI/PF/Paludisme Rapport final 2006 2011 Dakar: IntraHealth.

Mané B, Diop N, Termini N, Ramarao S, Clark H. 2012. Anneau vaginal à la Progestérone. Cartographie pays : Sénégal. Dakar : Population Council.

Mané B. Evolution de la Planification Familiale 1978-1986. 1994. In: Charbit Y et Ndiaye S. La population du Sénégal. Paris : Direction de la Prévision et de la Statistique (DPS), Centre d'Etudes sur les Populations Africaines et Asiatiques (CERPAA): p. 231-244.

Mbow FB, Ningue EAB, Diop N, Mané B, Ngouana R. 2015a. "La délégation des tâches dans le domaine de la planification familiale au niveau communautaire dans les pays du Partenariat de Ouagadougou : Expériences et leçons apprises pour une mise en œuvre effective - Présentation par pays". Dakar: Population Council.

Mbow FB, Ningue EAB, Diop N, Mané B, Ngouana R. 2015b. "La délégation des tâches dans le domaine de la planification familiale au niveau communautaire dans les pays du Partenariat de Ouagadougou : Expériences et leçons apprises pour une mise en œuvre effective -Rapport de synthèse". Dakar: Population Council.

Ministère de la Santé et de l'Action Sociale (MSAS). 2014. Lettre circulaire Nº08938/MSAS/DGS/CSC du 11 aout 2014 portant extension de l'offre initiale de contraceptifs injectables intramusculaires et souscutanés au niveau communautaire.

Ministère de la Santé et de la Prévention. 2010. Lettre circulaire Nº 007607/MSP/DSR du 26 juillet 2010 fixant les prix de cession des produits contraceptifs.

Ndiaye S, et Ayad M. 2006. Enquête Démographique et de Santé au Sénégal 2005. Calverton, Maryland, USA: Centre de Recherche pour le Développement Humain [Sénégal] et ORC Macro.

Partenariat de Ouagadougou. 2011. La planification familiale : l'Afrique de l'ouest francophone en mouvement. Un appel à l'action.

PATH. Janvier 2015. Recherche sur l'auto-injection de Sayana ${ }^{\circledR}$ Press. Seattle : PATH.

Pharmacie Nationale d'Approvisionnement (PNA). Décembre 2014. Modèle de Distribution Concertée (MoDisc) «jegesi naa ». Note Conceptuelle. Dakar : République du Sénégal.

Projet Initiative Sénégalaise de Santé Urbaine (ISSU). 2014a. Rapport de la capitalisation des approches du « paquet porteur » des interventions du projet ISSU. Dakar : IntraHealth International.

Réseau Siggil Jigeen. 2015a. «Le gain rapide. Cas de la commune de Pikine Djidah Thiaroye Kao ». Dakar. 
Réseau Siggil Jigeen. 2015b. Plaidoyer de budget avec le maire de la commune de Mbao. Dakar.

Réseau Siggil Jigeen. 2015c. Rapport trimestriel d’Advance Family Planning- Février Mars Avril 2015. Dakar.

Sidze EM, Lardoux S, Speizer IS, Faye CM, Mutua MM, Badji F. 2015. Accès et recours des jeunes femmes à la contraception: rôle des restrictions imposées par les prestataires en milieu urbain au Sénégal. Perspectives Internationales sur la Santé Sexuelle et Génésique. Numéro spécial de 2015, pp. 20-28. Doi: 10.1363/ FR02015.

The RESPOND Project. 2014. Reality Check experiences: Use of a program planning and advocacy tool for family planning initiatives. RESPOND Project Brief No. 22. July. New York: EngenderHealth (The RESPOND Project).

USAID-Sénégal. 2011. Expérience acquise de Programme de Santé Communautaire ChildFund/USAIDSénégal. Évaluation Finale - Programme de Santé Communautaire du Sénégal/ChildFund 2006-2011. Dakar : USAID Sénégal.

\section{Sites web consultés}

Bureau de l’OMS au Sénégal. Bulletin d’information du Bureau de l’OMS au Sénégal nº108, aout 2013.

Disponible sur http://www.afro.who.int/fr/senegal/press-materials/item/5842acc $\% \mathrm{C} 3 \% \mathrm{~A} 91 \% \mathrm{C} 3 \% \mathrm{~A} 9$ ration-de-la- $\mathrm{r} \% \mathrm{C} 3 \% \mathrm{~A} 9$ alisation-des-omd-relatifs- $\% \mathrm{C} 3 \% \mathrm{~A} 0-\mathrm{la}-$ roC3\%A9duction-de-la-mortalit \%C 3\%A9-maternelle-et-n\%C3\%A9onatale.html. Consulté le $14 / 10 / 2015$

Family Planning 2020a. Commitments. Senegal. Disponible sur http://www.familyplanning2020.org/commitments?entity type|=Country\&entity id $\rrbracket=88$. Consulté le $31 / 08 / 2015$.

Family Planning 2020b. Countries. Senegal. Disponible sur http://www.familyplanning2020.org/entities/88. Consulté le 31/08/2015.

Family Planning High Impact Practices. Pratiques à Haut Impact dans la Planification Familiale. 2014. Disponible sur https://www.fphighimpactpractices.org/sites/fphips/files/hip_list_fre.pdf. Consulté le $23 / 10 / 2015$.

Groupe pour l'Etude et l'Enseignement de la Population. GEEP en action. Disponible sur

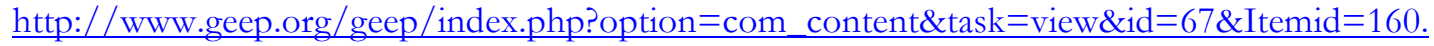
Consulté le 31/08/2015.

High-Impact Practices in Family Planning (HIP). 2014. Financing commodities and services: mobilizing resources to sustain current and future family planning demand. Washington, DC: USAID. Disponible sur: https://www.fphighimpactpractices.org/sites/fphips/files/hip_financing_fre.pdf. Consulté le 28/10/2015.

IntraHealth International. Newsroom. 2011. Une équipe dirigée par IntraHealth se lance dans un nouveau projet au Sénégal. Disponible sur http://www.intrahealth.org/page/une-quipe-dirige-par-intrahealthse-lance-dans-un-nouveau-projet-au-sngal. $\% 20$ Consulté $\% 201$ e $\% 2023 / 10 / 2015$. 
Partners in Population and Development Africa Regional Office. Le dividende démographique et le développement. Disponible sur http://www.ppdafrica.org/docs/policy/demo-f.pdf. Consulté le $\underline{22 / 10 / 2015}$.

\section{Présentations}

Daff Bocar Mamadou. Planification familiale au Sénégal. Amélioration de l'accès par les 3D. Conférence "Population, développement et planification familiale en Afrique de l'ouest francophone : l'urgence d'agir", Février 2011, Ouagadougou.

IntraHealth. Le TutoratPlus : la nouvelle génération, 2012.

Marie Stopes International. La Franchise Sociale MSI au Sénégal, Février 2015.

Marie Stopes international. Les équipes mobiles pour atteindre les populations défavorisées, Saint Louis, Avril 2015.

Marie Stopes International. Mobiliser les Jeunes pour un comportement sain et responsable, Mai 2014.

Projet Initiative Sénégalaise de Santé Urbaine (ISSU). 2014 b. «Synthèse des résultats de l'enquête à mi-parcours ». DSRSE, Atelier de restitution des résultats de l'enquête à mi-parcours, de l'évaluation de l'ISBC et du processus de capitalisation du « Paquet Porteur » du projet ISSU, Dakar, 24 juin 2014. 



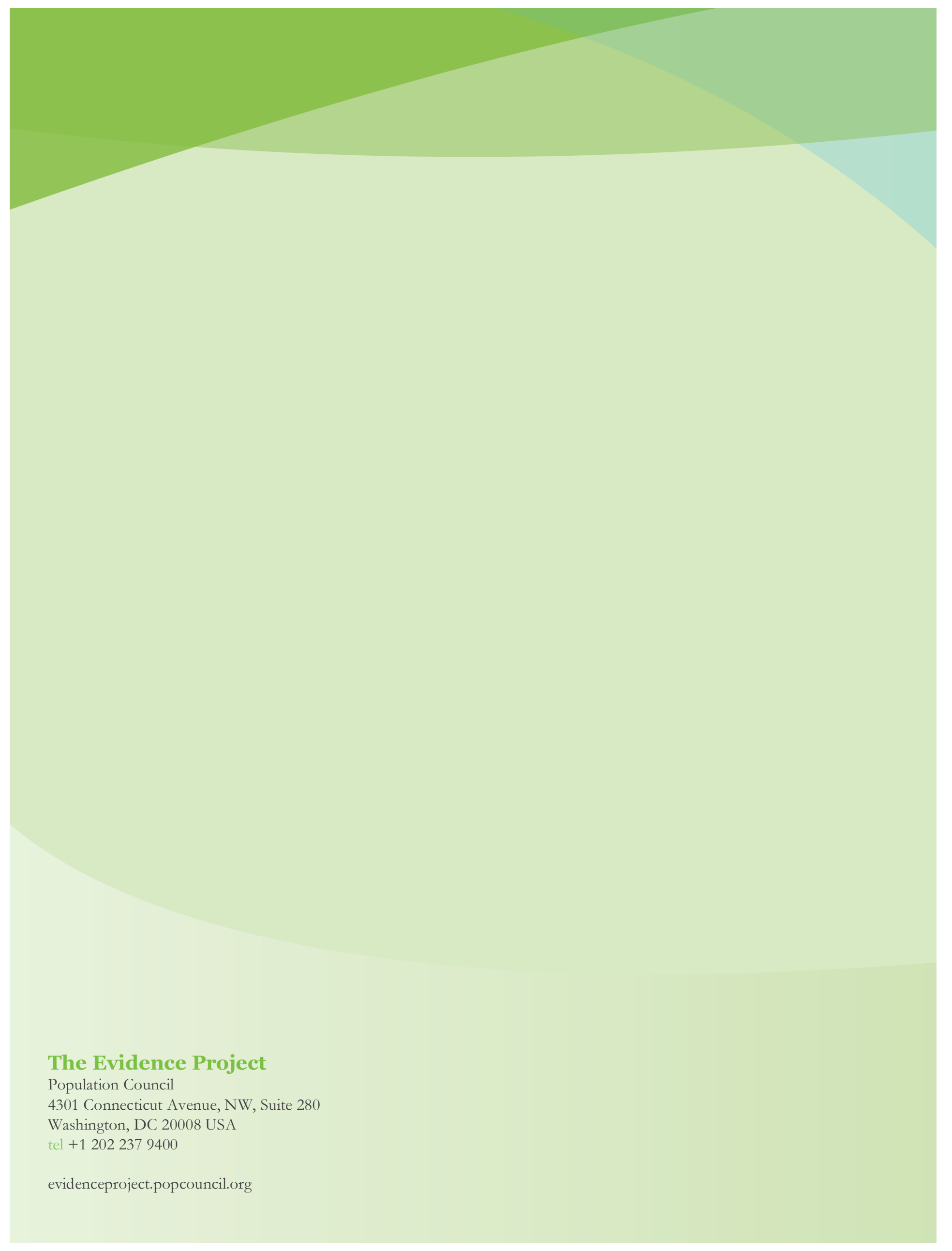

